\title{
Dynamics of crescent water wave patterns
}

\author{
By D. FRUCTUS ${ }^{1}$, C. KHARIF ${ }^{2}$, M. FRANCIUS $S^{2}$, \\ Ø. KRISTIANSEN ${ }^{1}$, D. CLAMOND ${ }^{1}$ AND J. GRUE \\ ${ }^{1}$ Mechanics Division, Department of Mathematics, University of Oslo. P.O. Box 1053 Blindern, \\ 0316 Oslo, Norway \\ ${ }^{2}$ Institut de Recherche sur les Phénomènes Hors-Equilibre, Technopôle de Château-Gombert, \\ 49 Rue Frédéric Joliot-Curie, B.P. 146, 13384 Marseille Cedex 13, France
}

(Received 1 July 2004 and in revised form 16 February 2005)

The nonlinear dynamics of three-dimensional instabilities of uniform gravity-wave trains evolving to crescent wave patterns is investigated numerically. A new mechanism of generation of oscillating horseshoe patterns is proposed and a detailed discussion on their occurrence in a water wave tank is given. It is suggested that these patterns are more likely to be observed naturally in water of finite depth. A critical wave steepness for the onset of three-dimensional wave breaking due to the nonlinear evolution of quintet resonant interactions corresponding to the phase-locked crescent-shaped structures (class II instability) is provided when the quartet resonant interaction (class I instability) is absent. The nonlinear coupling between quartet resonant interactions (class I instability) and quintet resonant interactions (class II instability) leading to three-dimensional breaking waves, as shown experimentally by Su \& Green (1984, 1985), is numerically investigated.

\section{Introduction}

The present study is devoted to three-dimensional crescent-shaped patterns occurring at the sea surface and more specifically to those emerging naturally from the instability of Stokes waves. These structures play an important role in wave breaking which for natural conditions are predominantly three-dimensional and cause transfer of momentum and energy between the ocean and the atmosphere. They may result from spontaneous instabilities of a two-dimensional water wave field. The stability of two-dimensional uniform Stokes wave trains has been studied for many decades. The pioneering authors were Lighthill (1965), Benjamin \& Feir (1967) and Zakharov (1968). Later McLean et al. (1981) and McLean (1982a) discovered a new kind of three-dimensional instability which, prior to this, had been suggested in a twodimensional study by Longuet-Higgins (1978). While pioneering works focused on instabilities resulting from a resonant interaction between four components of the wave field, McLean and co-authors investigated resonant five-wave interactions and suggested separating instabilities into two classes: class $\mathrm{I}(\mathrm{m})$ and class $\mathrm{II}(\mathrm{m})$ corresponding to $(2 m+2)$-wave interactions and $(2 m+3)$-wave interactions, respectively. In infinite depth McLean also considered the case $m=2$ that corresponds to resonant six- and seven-wave interactions. Our study is confined to interactions corresponding to $m=1$.

In water of infinite depth, it is well known that the two-dimensional modulational instability is dominant for small to moderate initial steepness $a k$ ( $a$ is the amplitude and $k$ the wavenumber) and evolves into a recurrence phenomenon (the Fermi-PastaUlam recurrence) for small initial wave steepness. This phenomenon is characterized 
by a series of modulation-demodulation cycles in which initially uniform wave trains become modulated and then demodulated until they are again uniform. Modulation is caused by growth of the two dominant sidebands of the Benjamin-Feir instability at the expense of the carrier wavenumber. Banner \& Tian $(1996,1998)$ predicted an energy exchange leading to breaking for initial steepness larger than a threshold value. Another kind of disturbance dominated by three-dimensional instabilities (see McLean 1982) exists and becomes dominant for larger values of $a k$. This instability may lead to the formation of horseshoe patterns evolving into three-dimensional spilling breakers. These three-dimensional patterns take the form of crescent-shaped perturbations riding on the basic waves.

$\mathrm{Su}$ et al. (1982) and Su (1982) performed a series of experiments on instabilities of Stokes waves of large steepness in deep water in a long tow tank and a wide basin. They observed three-dimensional structures corresponding to the nonlinear evolution of the dominant instability discovered by McLean et al. (1981). The initial twodimensional wave train of large steepness evolves into a series of three-dimensional spilling breakers, followed by a transition to a more or less two-dimensional wave train. Further experimental investigations were described by Melville (1982). The horseshoe patterns may also be produced in wave tank experiments in the presence of wind (Kusuba \& Mitsuyasu 1986). Generally, the two kinds of instability, namely the modulational instability and the crescent patterns which belong to class I and class II respectively, co-exist in the wave field. Depending on parameters such as the wave steepness of the initial Stokes wave and water depth, one can expect a competition to occur between the two classes of instability. Note that for shallow water cases and relatively moderate steepness, instability of a plane Stokes wave is dominated by class II (Francius \& Kharif 2005).

Su \& Green $(1984,1985)$ reported results of experimental investigations on a coupled interaction between the two classes of instability. They came to the conclusion that class I and II instabilities interact strongly during the evolution of wave trains with moderate initial steepness. They suggested that modulations produced by essentially two-dimensional instabilities (class I) were sufficient to trigger the predominantly three-dimensional instabilities (class II) that consequently limit the growth of the class I. Furthermore, they showed that this coupling may lead to the three-dimensional crescent-shaped breaking waves. Using the modified Zakahrov equation, Stiassnie \& Shemer (1987) examined the coupled evolution of class I and class II instabilities and found, in contrast to single class (I or II) evolution, that coupled behaviour was non-periodic. Except for very steep waves, they observed a dominance of class I interactions over those of class II and did not find the trigger mechanism.

Besides the three-dimensional patterns that are phase-locked with the plane Stokes waves, Collard \& Caulliez (1999) found oscillating three-dimensional crescent-shaped patterns in a wind wave facility. To isolate the three-dimensional pattern formation they used a thin plastic film to cover the water surface and a light wind to balance the damping effect due to the plastic film. The role of the film was twofold: to prevent the breaking of the waves and to eliminate the modulational instability. While the crescent patterns phase-locked to the basic wave are in staggered rows, the oscillating crescent patterns are aligned. These aligned patterns were observed for the first time by Skandrani (1996) who used a numerical code similar to that developed by Dommermuth \& Yue (1987). Later Kharif \& Pontier (2000) and Kharif, Pontier \& Skandrani (2000, personal communication) reported further numerical results on three-dimensional structures. Using direct numerical simulations Xue et al. (2001) 
investigated the nonlinear evolution of unstable three-dimensional disturbances of class II leading to $L_{2}$ and $L_{3}$ patterns as observed by Su. In addition to the $L_{2}$ and $L_{3}$ patterns, we consider $L_{1}$ patterns corresponding to the oscillating crescents. Very recently, Fuhrman, Madsen \& Bingham (2004) presented a numerical study of these patterns using the fully nonlinear and dispersive Boussinesq formulation. To explain wind wave horseshoe patterns seen on the sea surface Shrira, Badulin \& Kharif (1996) developed a simple model derived from the Zakharov equation, modified to include non-conservative effects. The model of the latter authors was revisited by Craig (2001). This aspect is also discussed in the review paper of Dias \& Kharif (1999).

The motivation of the paper is twofold: (i) to complete and extend previous studies on the dynamics of three-dimensional doubly periodic water wave patterns in infinite and finite depths, focusing particularly on the oscillating structures recently observed in a wave tank, (ii) to investigate numerically the nonlinear instability coupling leading to three-dimensional water wave breaking. The latter point was considered experimentally by Su \& Green $(1984,1985)$. In $\S 2$ the numerical model we use to solve the full set of water wave equations governing the nonlinear evolution of class I and class II instabilities is presented. Section 3 is devoted to the dynamics of crescent wave patterns due to class II instabilities in infinite depth and comparison with experimental results of Su (1982) and Collard \& Caulliez (1999). In $\S 4$ we focus our attention on the competition between crescent wave patterns and modulational instability, that is competition between different instabilities of class II and also between class I and class II instabilities. In $\S 5$ we discuss the effect of depth on the class competition. The case $k h=1$, where $h$ is the depth, is considered. In $\S 6$ is given an explanation for the selection mechanism that may be responsible for triggering the instability responsible of the occurrence of the oscillating patterns. Note that the corresponding resonant quintet is never the most linearly unstable perturbation of the class II. Numerical experiments are provided.

\section{Model for fully nonlinear simulations}

Breaking is strongly associated with splashing, turbulence, mixing and dissipation. This means that breaking of water waves is a turbulent two-phase flow. Theoretical modelling is very complex and the potential model we use is unable to capture these features. Herein, we consider numerical simulations up to the point where breaking occurs. This limitation is due to the Eulerian description used in the present model. A Lagrangian approach may allow calculations to continue up to the point at which the surface impacts on itself. Chen et al. (1999) provided numerical simulations describing plunging breakers including the splash-up phenomenon. They used a two-dimensional Navier-Stokes solver with a VOF technique. The method in use here is described in $\S 2.1$ and has been developed by Clamond \& Grue (2001) and Grue (2002). It was implemented and tested in Fructus et al. (2005).

\subsection{Notation and equations}

Let $\boldsymbol{x}=(x, y), z$ and $t$ be, respectively, the horizontal Cartesian coordinates, the upward vertical coordinate and the time; $z=0, z=-h$ and $z=\eta(\boldsymbol{x}, t)$ are, respectively, the equations of the still water level, of the impermeable horizontal bottom and of the impermeable free surface. Let also $\boldsymbol{v}=(\boldsymbol{u}, w)$ be the velocity field, where $\boldsymbol{u}=(u, v)$ and $w$ are the horizontal and vertical velocities, so that $\boldsymbol{v}=\operatorname{grad} \phi, \boldsymbol{u}=\nabla \phi$ and $w=\phi_{z} ; \phi$ is the velocity potential and $\nabla$ the horizontal gradient. We denote 
with tildes the quantities at the surface, e.g. $\widetilde{\phi}(\boldsymbol{x}, t)=\phi(x, z=\eta(\boldsymbol{x}, t), t)$. Note that $\widetilde{\boldsymbol{u}}=\widetilde{\nabla \phi} \neq \nabla \widetilde{\phi}=\widetilde{\boldsymbol{u}}+\widetilde{w} \nabla \eta$, and

$$
\widetilde{\boldsymbol{u}}=\frac{\nabla \widetilde{\phi}-V \nabla \eta+(\nabla \eta \times \nabla \widetilde{\phi}) \times \nabla \eta}{1+|\nabla \eta|^{2}}, \quad \widetilde{w}=\frac{V+\nabla \eta \cdot \nabla \widetilde{\phi}}{1+|\nabla \eta|^{2}},
$$

where $V=\phi_{n} \sqrt{1+|\nabla \eta|^{2}}, \phi_{n}$ being the outward normal derivative of $\phi$ at the surface.

At the impermeable free surface where the pressure is zero, the kinematic and dynamic conditions can be conveniently written as

$$
\eta_{t}-V=0, \quad \widetilde{\phi}_{t}+g \eta+\frac{1}{2} \widetilde{\boldsymbol{u}} \cdot \nabla \widetilde{\phi}-\frac{1}{2} \widetilde{w} V=0,
$$

due to $g$ being the acceleration of gravity.

The Laplace equation (resulting from incompressibility and irrotationality), together with the bottom impermeability, is solved exactly by means of a Green function and the method of images (Clamond \& Grue 2001; Grue 2002), i.e.

$$
\int_{S}\left(\frac{1}{\widetilde{r}}+\frac{1}{\widetilde{r}_{\mathrm{B}}}\right) \frac{\partial \phi^{\prime}}{\partial n^{\prime}} \mathrm{d} S^{\prime}=2 \pi \widetilde{\phi}+\int_{S} \widetilde{\phi}^{\prime} \frac{\partial}{\partial n^{\prime}}\left(\frac{1}{\widetilde{r}}+\frac{1}{\widetilde{\widetilde{r}_{\mathrm{B}}}}\right) \mathrm{d} S^{\prime},
$$

where $\widetilde{\phi}=\widetilde{\phi}(\boldsymbol{x}, t), \widetilde{\phi}^{\prime}=\widetilde{\phi}\left(\boldsymbol{x}^{\prime}, t\right), r^{2}=R^{2}+\left(z^{\prime}-z\right)^{2}$ and $r_{\mathrm{B}}^{2}=R^{2}+\left(z^{\prime}+z+2 h\right)^{2}$, with $R=|\boldsymbol{R}|$ where $\boldsymbol{R}=\boldsymbol{x}^{\prime}-\boldsymbol{x}$ (the horizontal distance between the source point and the field point). For non-overturning surfaces $\mathrm{d} S^{\prime}=\sqrt{1+\left|\nabla^{\prime} \eta^{\prime}\right|^{2}} \mathrm{~d} x^{\prime} \mathrm{d} y^{\prime}$, one thus obtains

$$
\begin{aligned}
& \int \frac{V^{\prime}}{\left(1+D^{2}\right)^{1 / 2}} \frac{\mathrm{d} \boldsymbol{x}^{\prime}}{R}+\int \frac{V^{\prime}}{\left(1+4 h D_{\mathrm{B}} R_{\mathrm{B}}^{-1}+D_{\mathrm{B}}^{2}\right)^{1 / 2}} \frac{\mathrm{d} \boldsymbol{x}^{\prime}}{R_{\mathrm{B}}} \\
= & 2 \pi \widetilde{\phi}+\int \frac{\widetilde{\phi}^{\prime}\left(\boldsymbol{R} \cdot \nabla^{\prime} \eta^{\prime}-\eta^{\prime}+\eta\right)}{\left(1+D^{2}\right)^{3 / 2}} \frac{\mathrm{d} \boldsymbol{x}^{\prime}}{R^{3}}+\int \frac{\widetilde{\phi}^{\prime}\left(\boldsymbol{R} \cdot \nabla^{\prime} \eta^{\prime}-\eta^{\prime}-\eta-2 h\right)}{\left(1+4 h D_{\mathrm{B}} R_{\mathrm{B}}^{-1}+D_{\mathrm{B}}^{2}\right)^{3 / 2}} \frac{\mathrm{d} \boldsymbol{x}^{\prime}}{R_{\mathrm{B}}^{3}},
\end{aligned}
$$

where $D=\left(\eta^{\prime}-\eta\right) / R, D_{\mathrm{B}}=\left(\eta^{\prime}+\eta\right) / R_{\mathrm{B}}, R_{\mathrm{B}}=\sqrt{R^{2}+4 h^{2}}$ and with the convenient brief notation

$$
\int \bullet \mathrm{d} \boldsymbol{x}^{\prime} \equiv \int_{-\infty}^{\infty} \int_{-\infty}^{\infty} \bullet \mathrm{d} x^{\prime} \mathrm{d} y^{\prime}
$$

The Fourier transform is written

$$
\widehat{\phi}(\boldsymbol{k}, t) \equiv \mathscr{F}\{\widetilde{\phi}(\boldsymbol{x}, t)\}=\int \widetilde{\phi}(\boldsymbol{x}, t) \mathrm{e}^{-\mathrm{i} \boldsymbol{k} \cdot \boldsymbol{x}} \mathrm{d} \boldsymbol{x} .
$$

In the deep water case, the terms involving $R_{\mathrm{B}}$ in equation (2.4) vanish. This equation is then inverted by means of Fourier transform and $\widehat{V}$ is decomposed into $\widehat{V}=\widehat{V}_{1}+\widehat{V}_{2}+\widehat{V}_{3}+\widehat{V}_{4}$. After some transformations, the contributions are given by

$$
\begin{aligned}
\widehat{V}_{1} & =k \widehat{\phi}, \\
\widehat{V}_{2} & =-k \mathscr{F}\left\{\eta V_{1}\right\}-\mathrm{i} \boldsymbol{k} \cdot \mathscr{F}\{\eta \nabla \widetilde{\phi}\}, \\
2 \pi \widehat{V}_{3}= & k \mathscr{F}\left\{\int \widetilde{\phi}^{\prime}\left[1-\left(1+D^{2}\right)^{-3 / 2}\right] \nabla^{\prime} \cdot\left[\left(\eta^{\prime}-\eta\right) \nabla^{\prime} R^{-1}\right] \mathrm{d} \boldsymbol{x}^{\prime}\right\}, \\
2 \pi \widehat{V}_{4}= & -\pi k \mathscr{F}\{\eta 2 \mathscr{F}-1\{k \mathscr{F}\{V\}\}-2 \eta \mathscr{F}-1\{k \mathscr{F}\{\eta V\}\}+\mathscr{F}-1\{k \mathscr{F}\{\eta 2 V\}\}\} \\
& +k \mathscr{F}\left\{\int V^{\prime} R^{-1}\left[1-\frac{1}{2} D^{2}-\left(1+D^{2}\right)^{-1 / 2}\right] \mathrm{d} \boldsymbol{x}^{\prime}\right\} .
\end{aligned}
$$


For the finite water depth case, all terms in the integral equation (2.4) have to be considered. This leads to a similar formulation where $\widehat{V}_{1}$ and $\widehat{V}_{2}$ are corrected by a factor $\tanh (k h)$ and where two additional integrals and some more convolution terms appear, see Grue (2002, §6) and Fructus et al. (2005).

The kernels of the inner integrals of $\widehat{V}_{3}$ and $\widehat{V}_{4}$ decay like $R^{-4}$ and $R^{-5}$, respectively. These integrals are evaluated over a very limited region of the $x$-plane. $V_{4}$ is determined implicitly and hence computed iteratively. For practical computations one iteration is shown to be sufficient. Moreover, the computations of the integrals are easily parallelizable. The implementation is done in Fortran 90 and allows computation on multiprocessor systems. For integrating the temporal system of equation (2.2), an analytical integration of the linear part of the system is first performed. The remaining nonlinear system is then solved via a six-stage fifth-order Runge-Kutta scheme with an embedded fourth-order scheme for the time-stepping control. To avoid aliasing errors, we use an anti-aliasing technique which is based on zeros padding. It is important to stress that this is the only method which is used to avoid numerical instabilities, i.e. we do not use a smoothing technique for instance. This yields a method which is very stable and accurate.

In order to emphasize the efficiency and the potential of the method, we have run test cases where the propagation of an initial Stokes wave in infinite depth was considered (since this is the initial background wave field we will mainly consider in this paper). The present study use as examples initial Stokes waves of steepness $a k$ mainly in a range between 0.1 to 0.3 . For this range, the method developed by Fenton (1988) is used to compute the steady waves. In consequence we first test the validity of the model for an initial steepness of 0.3 in a run where the wave is propagated forward in time during 1000 periods of the Stokes wave. To check the reversibility of the scheme we then perform a backward propagation (in time) from the last computed result. The total energy (defined by $\left.2 E=\int\left(\eta_{t} \widetilde{\boldsymbol{\phi}}+g \eta^{2}\right) \mathrm{d} \boldsymbol{x}\right)$ as well as the evolution of the phase shift are monitored during the simulation. The wave field (considering two wavelengths in the propagation direction) is discretized over $64 \times 64$ collocation points per period (meaning that the first seven harmonics of the Stokes wave are resolved). The resulting simulation (not shown here) shows that the phase shift increases linearly with time, reaching $18^{\circ}$ after 1000 periods of propagation. The phase shift then decreases to zero during the backward integration. Simultaneously the relative energy error $(E(t)-E(0)) / E(0)$ is of order $10^{-5}$ (and is almost constant over the time span of the simulation). An important point is that no trend is observed in the evolution of the energy (even after 2000 periods of propagation). The absence of a trend is crucial for the reversibility of the scheme and emphasizes the fact that no smoothing techniques have been used (those usually have a dramatic effect on the energy conservation!). The initial condition is perfectly recovered, meaning that the errors due to the time integrator, the computation of the integrals and the round-off errors are insignificant over the time scale considered.

We have extended the numerical tests of the method a bit further: waves with steepness up to $a k=0.4$ (numerical simulations with $a k=0.38$ and $a k=0.4$ have been performed). Since Fenton's method becomes less efficient when the steepness becomes too high, we used the iterative method developed by Longuet-Higgins (1985) to compute these very steep waves. Since the new test is aimed at showing that the model can deal with large values of the steepness and still be highly stable, we decreased the values of numerical parameters to do faster simulations. The numerical experiments were then performed over 100 periods in a back and forth time simulation 
when the parameter determining the number of iterations in the evaluation of $V_{4}$ was decreased from 3 to 1 (which in the previous case led to a phase shift of about $10^{\circ}$ after 100 periods). The relative error in the energy was observed to be always lower than $10^{-3}$ (no trend was observed) while the phase shift had a maximum value of about $20^{\circ}$ after 100 periods. As previously the initial condition was accurately recovered and the solution was very stable during the simulations without requiring any smoothing.

Another important issue is the computational time. We checked the time simulation on a single processor machine equipped with a $1.3 \mathrm{GHz}$ Pentium P4-M processor. For the computations corresponding to figures 3 and 5 (see $\S 3.2 .1$ below) three iterations were used for the computation of $V_{4}$ (as in all the other numerical simulations done in the paper). The total computational time for the two simulations was around $5500 \mathrm{~s}$.

This demonstrates that the method is fast and accurate. The total energy is very well conserved: this is due to the fact that no smoothing is used. The procedure is highly stable, however.

\subsection{Onset of breaking identification}

Wave breaking is characterized by the overturning of the free surface. During this process the slope of the surface becomes infinite, leading numerically to a spread of energy into high wavenumbers. This local steepening is characterized by a numerical blow-up (for methods dealing with an Eulerian description of the flow). Numerical blow-up is not necessarily a breaking criterion, however. Several kinematic and dynamic criteria for wave breaking have been suggested such as maximum horizontal velocity at the crest compared to the speed of the crest or maximum vertical acceleration or potential energy/total energy exceeding a given threshold. The works of Dold \& Peregrine (1986) and Banner \& Tian (1998) suggested that breaking may occur when the relative growth rates of the mean momentum and energy density, at their envelope maxima, are sustained at a given threshold of 0.2. Clearly, no simple breaking criteria exist. This is true even for a two-dimensional wave field.

Our purpose is not to provide a parameter criterion for the onset of wave breaking. We merely want to check that the numerical blow-up we observe, in the cases where it occurs, corresponds to an effective breaking of the wave. In order to check that, all the simulations leading to breaking are performed for several refinements of the grid. In addition, we perform a test based on the Banner \& Tian (1998) (and Dold \& Peregrine 1986) work. We consider an initial modulated wave group with five waves in the modulation. Following Banner \& Tian $(1996,1998)$, two small linearly unstable spectral sidebands are superimposed on the initial free-surface profile. According to their investigation a periodic transfer of energy between the main frequency and the sidebands (Fermi-Pasta-Ulam recurrence phenomenon) is observed for an initial wave steepness $(a k)_{0}=0.11$. For higher values of the steepness wave breaking is observed. We have repeated the numerical experiments of Banner \& Tian with $(a k)_{0}=0.11$ and $(a k)_{0}=0.1125$. Figure 1 shows the free-surface profiles of these two simulations at the same dimensionless time $t / T_{0}$ just before breaking of the simulation corresponding to an initial steepness $(a k)_{0}=0.1125$. The simulation corresponding to an initial steepness $(a k)_{0}=0.11$ does not lead to breaking but to a series of modulation/demodulation cycles as illustrated in figure 2 which shows the energy transfer between the fundamental mode and the sidebands for both cases. The energy $E$ is the square of the amplitude of satellite modes while $E_{0}$ is the square of the amplitude of the fundamental mode of the unperturbed Stokes wave. Our method cannot describe the jet formation, nevertheless it can predict whether 

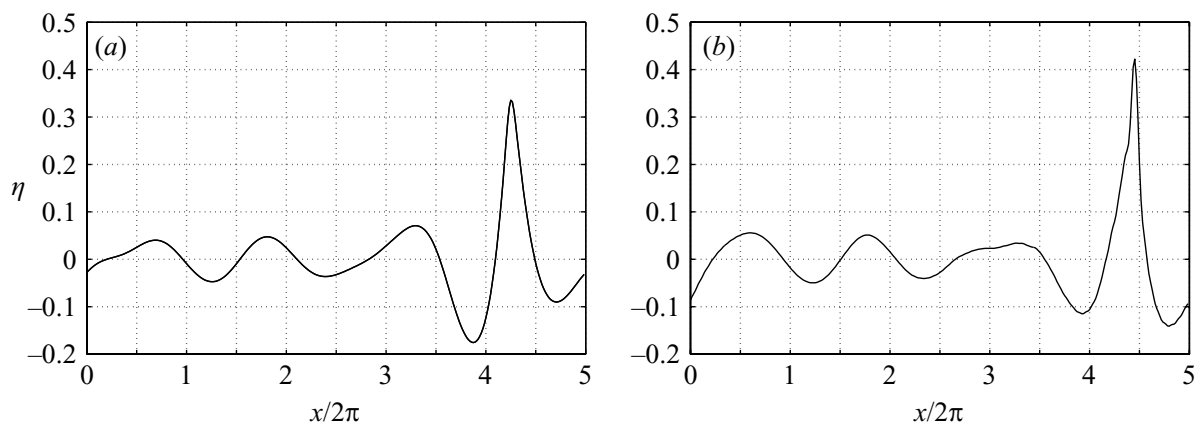

FIGURE 1. Free-surface profiles of an evolved wave group with five waves in the modulation. To the initial Stokes wave has been added two small sidebands at $t / T_{0}=0$. ( $a$ ) An evolved wave group with initial steepness $(a k)_{0}=0.11$ at $t / T_{0}=123$, in this case the evolution is periodic. (b) An initial steepness $(a k)_{0}=0.1125$ at $t / T_{0}=123$, this case leads to breaking.
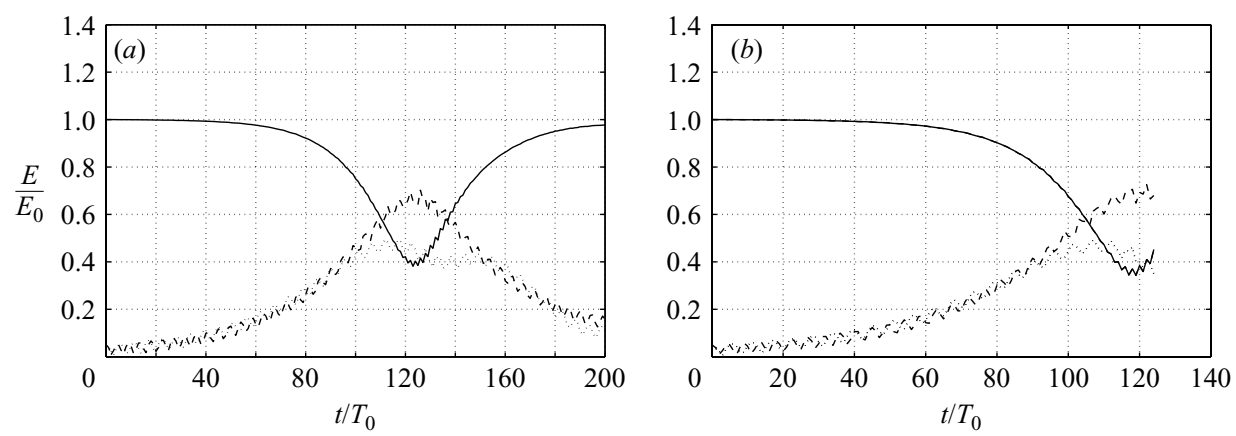

FIGURE 2. Normalized energy evolution of the fundamental mode and satellites: $(k, 0)$ (solid line), $(0.8 k, 0)$ (dashed line) and $(1.2 k, 0)$ (dotted line). $(a)$ An initial steepness $(a k)_{0}=0.11$ showing the periodic modulations. $(b)$ An initial steepness $(a k)_{0}=0.1125$ showing the numerical blow-up of the simulation corresponding to breaking.

an initial uniform Stokes wave train will evolve or not into a breaking wave. The numerical blow-up we have observed, when it is independent of the grid refinement, corresponds physically to the onset of breaking.

\section{Dynamics of crescent wave patterns on deep water}

\subsection{Initialization}

The classical water wave problem consists of solving, for the velocity potential, $\phi$, and the elevation of the free surface, $\eta$, the Laplace equation in the flow domain with nonlinear boundary conditions at the free surface and a bottom condition. It is well known that this problem admits two-dimensional periodic solutions in the form of travelling gravity waves (Stokes waves). Let us denote $\bar{\eta}$ and $\bar{\phi}$ the elevation and the potential of the Stokes wave of fundamental wavenumber $k$ and phase speed $c$ (which in what follows below are evaluated using the method developed by Fenton 1988).

Stokes waves are unstable to perturbations. Let $\eta(x, y, t)=\bar{\eta}(x, t)+\eta^{\prime}(x, y, t)$ and $\phi(x, y, z, t)=\bar{\phi}(x, z, t)+\phi^{\prime}(x, y, z, t)$ with $\eta^{\prime}(x, y, t) \ll 1$ and $\phi^{\prime}(x, y, z, t) \ll 1$.

To calculate the infinitesimal disturbances $\eta^{\prime}$ and $\phi^{\prime}$, the water wave equations are linearized about $\bar{\eta}$ and $\bar{\phi}$. Non-trivial solutions of the linearized problem are sought 
in the form

$$
\begin{gathered}
\eta^{\prime}(x, y, t)=\mathrm{e}^{s t+\mathrm{i} p k(x-c t)+\mathrm{i} q k y} \sum_{n=-\infty}^{n=+\infty} a_{n} \mathrm{e}^{\mathrm{i} n k(x-c t)}+\text { c.c. }, \\
\phi^{\prime}(x, y, z, t)=\mathrm{e}^{s t+\mathrm{i} p k(x-c t)+\mathrm{i} q k y} \sum_{n=-\infty}^{n=+\infty} b_{n} \mathrm{e}^{\mathrm{i} n k(x-c t)+\gamma_{n} z}+\text { c.c. },
\end{gathered}
$$

with $\gamma_{n}=k \sqrt{(p+n)^{2}+q^{2}}$, where $p$ and $q$ are arbitrary real numbers and c.c. denotes the complex conjugate. The truncated series are substituted into the linearized equations to give an eigenvalue problem. The eigenvalues are the values of $s$ such that there is a non-trivial solution with time-dependence $\mathrm{e}^{s t}$. Instability corresponds to $\operatorname{Re}(s)>0$. Once the steepness $a k$ and the real numbers $p$ and $q$ are fixed, the spectrum and the corresponding eigenfunctions are computed until convergence is obtained by increasing the order of truncation (for more details see Kharif \& Ramamonjiarisoa 1988, 1990). In order to produce the occurrence of the crescent wave patterns observed in experiments, the initial condition is a Stokes wave of steepness $a k$ disturbed by an unstable three-dimensional perturbation (instability of class II). The normalized amplitude, $\epsilon$, of the perturbation relative to the Stokes wave amplitude is initially taken of order $10^{-2}$. For simplicity and without loss of generality we set the fundamental wave-vector of the Stokes wave $\boldsymbol{k}_{0}=(k, 0)=(1,0)$. The wave-vectors of the dominant modes of the unstable perturbation of class II are $\boldsymbol{k}_{1}=\left(k_{x}, k_{y}\right)=(1+p, q)$ and $\boldsymbol{k}_{2}=\left(k_{x}, k_{y}\right)=(2-p,-q)$ while for instability of class I they are $\boldsymbol{k}_{1}=\left(k_{x}, k_{y}\right)=(1+p, q)$ and $\boldsymbol{k}_{2}=\left(k_{x}, k_{y}\right)=(1-p,-q)$.

For numerical simulations we consider a wave tank of length $L=2 \pi N$ and width $W=2 \pi M / q$, where $N$ and $M$ are integers. For a given number of wavelengths $(N)$ of the Stokes wave in the numerical tank, Benjamin-Feir instabilities may or not be captured in the computational domain. This means that Benjamin-Feir instabilities may be avoided so as to reproduce experimental conditions when a plastic film is used.

We performed convergence tests resolving up to 32 harmonics of the Stokes waves in the $x$-direction and 32 harmonics of the perturbation in the transverse direction. We found that, most of the time (for small to moderate initial steepness as considered here), eight harmonics were sufficient in both directions. Higher wave modes were negligible throughout the time simulation.

The number of grid points in the two horizontal dimensions are chosen such that for all simulations at least eight harmonics of the Stokes waves in the main propagation direction and at least eight harmonics of the perturbation in the transverse direction, are discretized. The numerical scheme needs no smoothing but uses the zeros-padding technique to perform de-aliased computations of the convolution sums. We choose to take the padding factor equal to two in order to be able to resolve cubic nonlinearity without aliasing. This means that the numerical grid in use has, at least, $32 \mathrm{~N}$ points in the $x$-direction and $32 M$ points in the $y$-direction.

\subsection{Evolution of isolated class II instabilities}

In this section we consider the nonlinear evolution of an initial Stokes wave train of fundamental wave-vector, $\boldsymbol{k}_{0}$, and frequency, $\omega_{0}$, perturbed by a single instability corresponding to given values of $p$ and $q$. The perturbation is chosen to belong to class II instability. It is known, however, that for an initial steepness $(a k)_{0}<0.314$ of the unperturbed Stokes waves the most unstable perturbation is of class I. Herein we impose that the class I instability must not develop. This may be justified by the fact 
that in experimental setups, the use of a plastic film on the free surface can play a similar role, cancelling or attenuating the modulational instability. Note that in finite depth the strength of the dominant instabilities of class I and class II may be of the same order when the wave is moderately steep. Competition between instabilities of class II and class I is considered in $\S 4.2$.

The numerical discretization is chosen in order to avoid the presence of unstable spectral components which belong to class I. This means that for practical computations involving Stokes wave trains of small to moderate initial steepness we set $N=2$.

The evolution of class II instabilities have been the topic of many studies. Experimental investigations (Su 1982; Melville 1982) as well as theoretical (Shrira et al. 1996) and numerical studies (Xue et al. 2001; Fuhrman et al. 2004) provide a good understanding of the underlying phenomenon responsible for the formation of crescent wave patterns. Two satellites of wave-vectors $\boldsymbol{k}_{1}$ and $\boldsymbol{k}_{2}^{*}$ and frequency $\omega_{1}$ and $\omega_{2}^{*}$, may resonate with the fundamental of the Stokes wave, $\boldsymbol{k}_{0}$, in a quintet interaction if the following resonance condition is fulfilled:

$$
\left.\begin{array}{rl}
\boldsymbol{k}_{1}+\boldsymbol{k}_{2}^{*} & =3 \boldsymbol{k}_{0} \\
\omega_{1}+\omega_{2}^{*} & =3 \omega_{0} .
\end{array}\right\}
$$

This quintet interaction leads to the formation of crescent wave patterns.

Let us consider an initial perturbation with dominant wave-vectors $\boldsymbol{k}_{1}=(1+1 / n, q)$ and $\boldsymbol{k}_{2}^{*}=(2-1 / n,-q)$. This means that $p=1 / n$ where the integer $n \geqslant 2$. If the frequency condition is satisfied the quintet interaction is resonant. The amplitude of the components of the perturbation corresponding to these wave-vectors increases exponentially as time increases and then saturates owing to nonlinearity to reach a maximum when the amplitude of the fundamental is minimum. Following $\mathrm{Su}$ (1982), the nonlinear development of this instability leads to the formation of $L_{n}$ patterns.

The most unstable perturbation of class II corresponds to $p=\frac{1}{2}$. The corresponding $L_{2}$ pattern is phase-locked to the unperturbed Stokes waves. This means that the corresponding horseshoe pattern travels with the same speed as the undisturbed wave. Many observations of such patterns have been reported.

Another interesting three-dimensional pattern was generated experimentally by Collard \& Caulliez (1999). They observed the development of the unstable mode corresponding to $p=0$. As pointed out by McLean (1982a) there is an artificial degeneracy in the choice of $p$ which can be removed by restricting $p$ to the range $0 \leqslant p<1$. So the structures corresponding to $p=0$ correspond also to $n=1$. For that case two pairs of dominant components fulfil (3.2). The definition by $\mathrm{Su}$ can be used for $n=1$. Collard \& Caulliez named these $L_{1}$ structures 'oscillating horseshoe' patterns.

In order to check the quintet interaction conditions (3.2), we compute, for each simulation, the evolution of the magnitude of the Fourier transform of the perturbed wave field $\left|\mathscr{F}(\eta)\left(k_{x}, k_{y}, t\right)\right|$. We investigate simultaneously the frequency spectrum, $\omega\left(k_{x}, k_{y}, t\right)$. This is obtained from the Fourier transform of the perturbed wave field from $\mathscr{F}(\eta)=|\mathscr{F}(\eta)| \exp (\mathrm{i} \chi)$ and $\omega\left(k_{x}, k_{y}, t\right)=\partial \chi / \partial t$. This means that we can check the frequency evolution for each individual mode $\left(k_{x}, k_{y}\right)$.

\subsubsection{Evolution leading to breaking and comparison with available experiments}

First, we investigate the case $n=2$ ( or $p=\frac{1}{2}$ ) which corresponds to the phaselocked $L_{2}$ patterns that were observed experimentally by $\mathrm{Su}$ (1982), Melville (1982), Kusuba \& Mitsuyasu (1986) and Collard \& Caulliez (1999). In the Su and Melville 

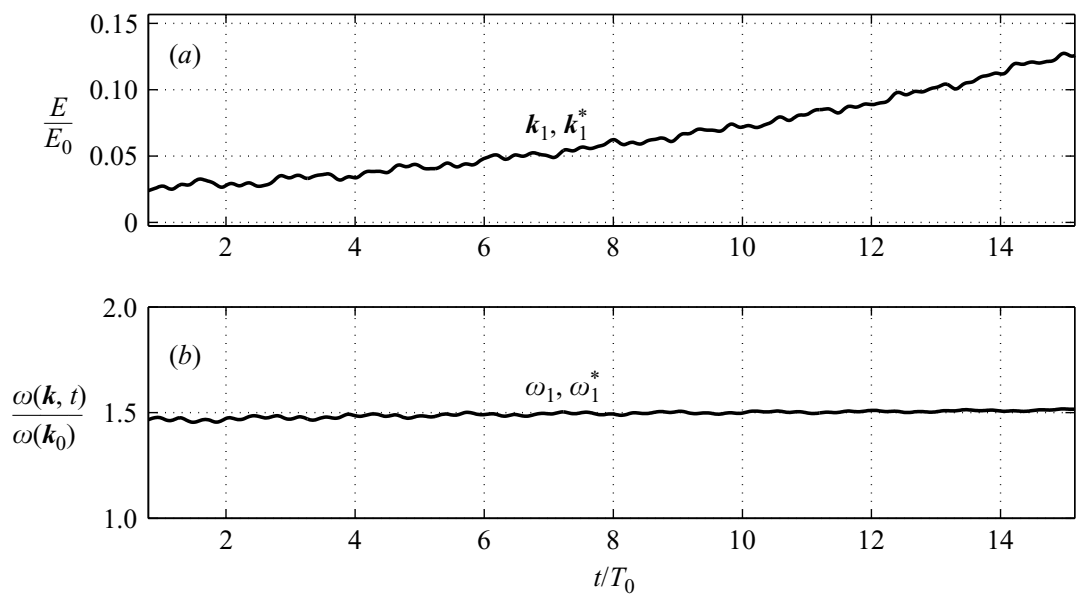

FIGURE 3. (a) Normalized energy evolution of the dominant modes $\boldsymbol{k}_{1}=\left(\frac{3}{2}, \frac{4}{3}\right)$ and $\boldsymbol{k}_{1}^{*}=$ $\left(\frac{3}{2},-\frac{4}{3}\right)$. (b) Evolution of the frequency $\omega$. The initial perturbation corresponds to $(p, q)=\left(\frac{1}{2}, \frac{4}{3}\right)$, $(a k)_{0}=0.2985$ and $\epsilon=0.05$.

experiments the initial wave steepness was $(a k)_{0} \approx 0.31$. For this value of the steepness the class II instability overtakes class I instability and it is found that the development of those instabilities leads to breaking through the formation of spilling breakers.

We compute numerically the nonlinear evolution and growth of crescent structures up to the occurrence of the wave breaking as defined in $\$ 2.2$. We compare the simulated wave fields prior to overturning of the wave with observations that are available. Note that experimental observations deviate from numerical simulations in the respect that in experiments a steady state is reached. Within the framework of our numerical model, a further growth of the amplitude is limited by the breaking of the waves.

It is expected that the growth of the dominant modes $\boldsymbol{k}_{1}=(1+p, q)=(3 / 2, q)$ and $\boldsymbol{k}_{2}^{*}=(2-p,-q)=(3 / 2,-q)$ of the instability will be observed. The three-dimensional patterns propagate with the phase speed of the basic wave. Numerical simulations for an initial perturbation with $(p, q)=(1 / 2,4 / 3)$ are performed taking $(a k)_{0}=0.2985$, and $\epsilon=0.05$. Figure 3 displays the normalized energy and frequency evolution of the dominant modes, over 16 periods of the unperturbed Stokes wave. As expected the quintet interaction predominates and the wave-vectors and frequencies of the modes responsible for this resonant interaction satisfy condition (3.2).

We make a direct comparison with available experimental data just before the breaking stage is reached. Su defined six geometric parameters (see figure 4) and seven ratio-aspect parameters to describe the $L_{2}$ patterns. Table 1 gives a comparison between Su's experimental results and our numerical computations. Our results show a maximal relative difference from the observations of less than $1 \%$ for four of the parameters and $6 \%$ for the remaining parameters. This means that the final stage of the computed steady horse shoe patterns corresponds to the experimentally observed structures, both in amplitude and wavelength. Figure 5(a) displays the free-surface elevation of the wave field prior to breaking that corresponds to the horseshoe patterns as obtained experimentally.

Few experimental studies have been devoted to the less understood $L_{1}$ pattern. To our knowledge Collard \& Caulliez (1999) are the only authors to have observed this pattern experimentally, up to now. They noted that more understanding is 


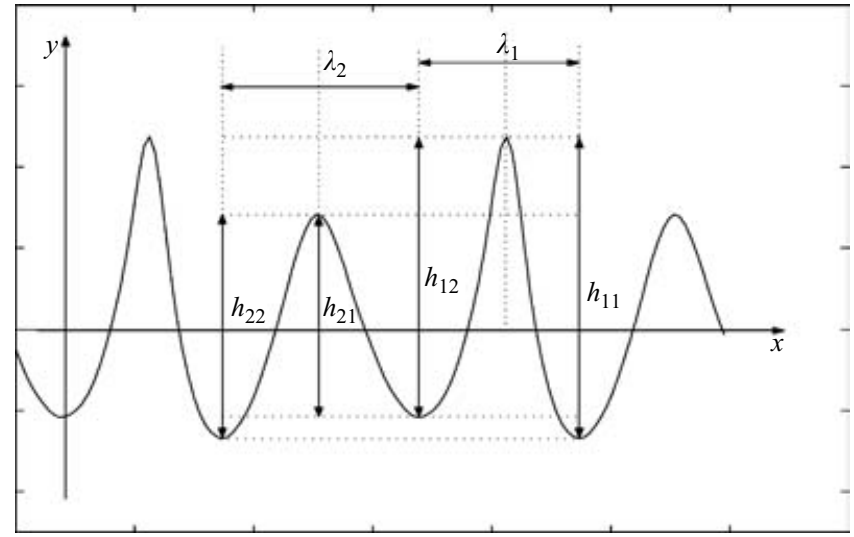

Figure 4. Definition of Su's geometric parameters.
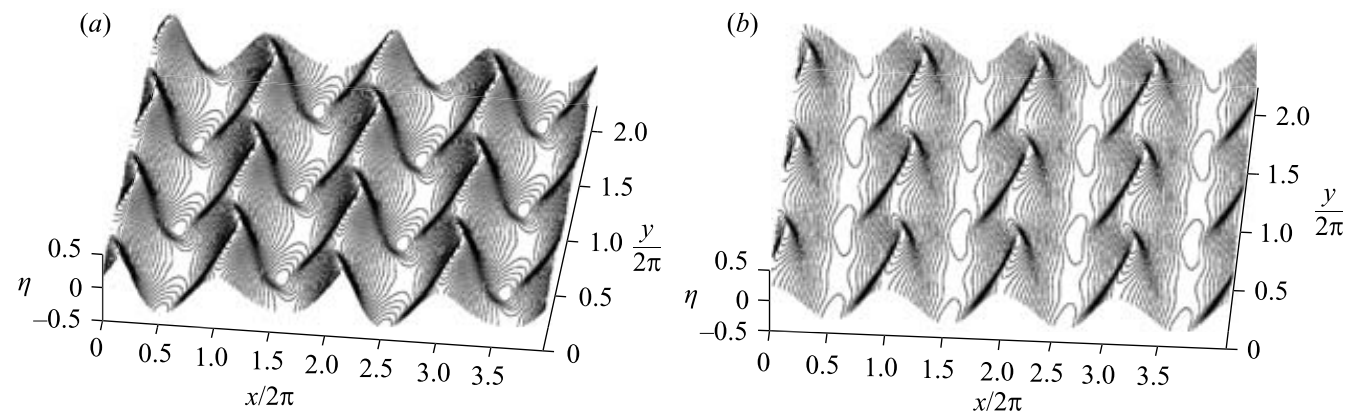

FIGURE 5. Free surface elevation corresponding to $(a)$ figure 3 at $t / T_{0}=18$ and $(b)$ figure 5 at $t / T_{0}=23$.

\begin{tabular}{lccccccc}
\hline & $\lambda_{2} / \lambda_{1}$ & $h_{11} / h_{12}$ & $h_{21} / h_{22}$ & $h_{11} / h_{21}$ & $h_{22} / h_{11}$ & $\frac{h_{11}+h_{12}}{h_{22}+h_{21}}$ & $S_{\max }$ \\
& 1.28 & 1.10 & 0.88 & 1.66 & 0.68 & 1.49 & 0.65 \\
$\begin{array}{l}\text { Su results } \\
\text { Present results }(a k=0.2985)\end{array}$ & 1.28 & 1.11 & 0.88 & 1.56 & 0.73 & 1.38 & 0.66 \\
$\begin{array}{l}\text { Present results }(a k=0.33) \\
\quad t / T_{0}=8\end{array}$ & 1.05 & 1.09 & 0.91 & 1.27 & 0.86 & 1.16 & 0.46 \\
$\begin{array}{c}\text { Present results }(a k=0.33) \\
\quad t / T_{0}=9\end{array}$ & 1 & 1.08 & 0.91 & 1.31 & 0.84 & 1.20 & 0.61 \\
$\begin{array}{c}\text { Present results }(a k=0.33) \\
t / T_{0}=10.75\end{array}$ & 1.28 & 1.12 & 0.85 & 1.53 & 0.76 & 1.33 & 0.67
\end{tabular}

TABLE 1. Comparison of characteristic crescent wave geometric parameters for which a quasi-steady state is assumed to be observed for $a k=0.2985$ and evolution of those parameters for $a k=0.33$ until the same state at $t / T_{0}=10.75$.

required to explain its generation. Skandrani (1996) emphasized the existence of such aligned patterns in his numerical simulations. Recently Fuhrman et al. (2004) provided numerical simulations of these structures. We choose the initial perturbation corresponding to $(p, q)=\left(0, \frac{4}{3}\right)$ and $\epsilon=0.05$, which is a class II instability for the initial wave steepness considered $(a k)_{0}=0.2985$. The magnitude of the Fourier transform 

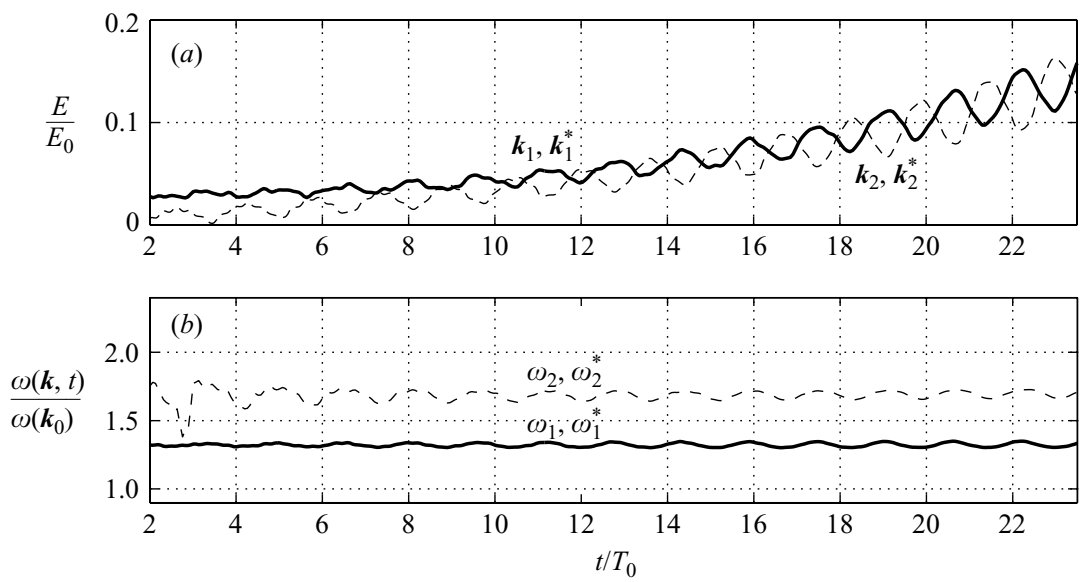

FIGURE 6. (a) Normalized energy evolution of the dominant modes $\boldsymbol{k}_{1}=\left(1, \frac{4}{3}\right), \boldsymbol{k}_{1}^{*}=\left(1,-\frac{4}{3}\right)$, $\boldsymbol{k}_{2}=\left(2, \frac{4}{3}\right)$ and $\boldsymbol{k}_{2}^{*}=\left(2,-\frac{4}{3}\right)$. (b) Evolution of the frequency $\omega$. The initial perturbation corresponds to $(p, q)=\left(0, \frac{4}{3}\right),(a k)_{0}=0.2985$ and $\epsilon=0.05$.

of the perturbed wave field, $|\mathscr{F}(\widehat{\eta})|$, shows a pronounced growth of the modes with wave-vectors $\boldsymbol{k}_{1}=\left(1, \frac{4}{3}\right)$ and $\boldsymbol{k}_{2}^{*}=\left(2,-\frac{4}{3}\right)$ which satisfy $\boldsymbol{k}_{1}+\boldsymbol{k}_{2}^{*}=3 \boldsymbol{k}_{0}$. Figure 6 displays the energy and frequency evolution over 25 periods of the main components of the wave field. Note that the energy corresponding to higher-order wave components remains small as seen on figure $6(a)$.

Figure $6(b)$ shows that the frequencies become (almost) constant during the simulation. Moreover, it is seen that the frequency resonant condition is satisfied: $\omega\left(\boldsymbol{k}_{1}\right)+\omega\left(\boldsymbol{k}_{2}^{*}\right) \simeq 3 \omega_{0}$.

The temporal evolution of the amplitude of the modes $\boldsymbol{k}_{1}^{*}=\left(1,-\frac{4}{3}\right)$ and $\boldsymbol{k}_{2}=\left(2, \frac{4}{3}\right)$ happens to be identical of that of $\boldsymbol{k}_{1}=\left(1, \frac{4}{3}\right)$ and $\boldsymbol{k}_{2}^{*}=\left(2,-\frac{4}{3}\right)$ respectively. It is not one resonant quintet interaction that results but two described by the following relations:

$$
\left.\begin{array}{ll}
\boldsymbol{k}_{1}+\boldsymbol{k}_{2}^{*}=3 \boldsymbol{k}_{0}, & \boldsymbol{k}_{1}^{*}+\boldsymbol{k}_{2}=3 \boldsymbol{k}_{0}, \\
\omega\left(\boldsymbol{k}_{1}\right)+\omega\left(\boldsymbol{k}_{2}^{*}\right)=3 \omega_{0}, & \omega\left(\boldsymbol{k}_{1}^{*}\right)+\omega\left(\boldsymbol{k}_{2}\right)=3 \omega_{0} .
\end{array}\right\}
$$

One can also note that these modes satisfy the following relations:

$$
\left.\begin{array}{l}
\boldsymbol{k}_{1}+\boldsymbol{k}_{2}^{*}=\boldsymbol{k}_{1}^{*}+\boldsymbol{k}_{2} \\
\omega\left(\boldsymbol{k}_{1}\right)+\omega\left(\boldsymbol{k}_{2}^{*}\right)=\omega\left(\boldsymbol{k}_{1}^{*}\right)+\omega\left(\boldsymbol{k}_{2}\right) .
\end{array}\right\}
$$

This resonant interaction between $\boldsymbol{k}_{1}, \boldsymbol{k}_{2}^{*}, \boldsymbol{k}_{1}^{*}$ and $\boldsymbol{k}_{2}$ explains the behaviour of their amplitudes. The oscillations depicted in figure 5(a) are due to the transfer of energy among these components.

Figure $5(b)$ shows the free-surface elevation of the wave field prior to breaking that corresponds to the oscillating horseshoe pattern as obtained experimentally by Collard \& Caulliez (1999). Here the three-dimensional structures are periodically shifted by one-half of the transverse wavelength, as was observed in the experiments. The period of oscillation is that of the perturbations $\left\{\boldsymbol{k}_{1}, \boldsymbol{k}_{2}^{*}\right\}$ and $\left\{\boldsymbol{k}_{1}^{*}, \boldsymbol{k}_{2}\right\}$ respectively, in the moving frame of reference, i.e. approximately $2.7 T_{0}$, where $T_{0}$ is the fundamental period of the Stokes wave. Collard \& Caulliez reported oscillations of the patterns with a period of approximately three times $T_{0}$. In their experiments they considered wave steepness of about $(a k)_{0}=0.15$, whereas we show results for $(a k)_{0}=0.2985$. This 
(a)

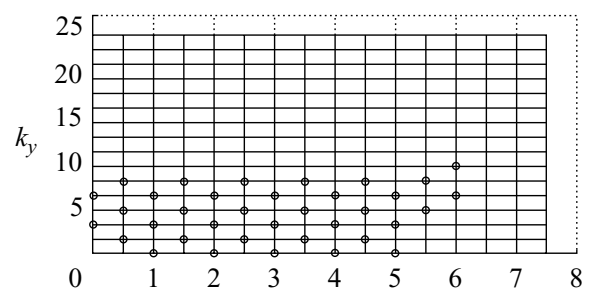

(b)

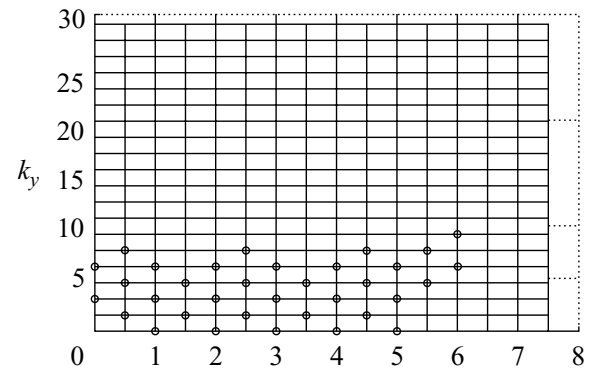

FIGURE 7. Excited modes for two different transverse grid resolutions in a simulation with $(a k)_{0}=0.2$ and dominant modes $\boldsymbol{k}_{1}=\left(\frac{3}{2}, 1.53\right)$ and $\boldsymbol{k}_{1}^{*}=\left(\frac{3}{2},-1.53\right)$. These spectra correspond to the end of the phase of increase of the satellites when the surface elevation shows fully developed horseshoe patterns. (a) 16 modes in the transverse direction are solved with 64 collocation points and (b) 20 modes in the transverse direction are solved with 80 collocation points.

difference does not affect the results (see $\S 3.2 .3$ ) presented here qualitatively but is essential for the selection mechanism, as demonstrated below.

Other patterns, such as $L_{3}$ and $L_{4}$ have been studied, however. The dynamics is similar and the quintet involving $\boldsymbol{k}_{1}=(1+1 / n, q)$ and $\boldsymbol{k}_{2}^{*}=(2-1 / n,-q)$ is obvious in each case.

Further results to confirm the excellent agreement with Su's experimental results are given in table 1 where we present the evolution of the parameters in a simulation where $a k=0.33$ (and $\epsilon=0.05$ ). This value, being considered closer to the experimental setup, corresponds to the numerical simulation by Fuhrman et al. (2004). All the geometrical parameters converge toward values which are close to the experimental ones. We note here as well that we obtain breaking at the last time step presented in table 1. This corresponds to a normalized time $t / T_{0}=10.75$, while Fuhrman et al. (2004) reported a breakdown of their simulation at a slightly larger time (at $t / T_{0}=11.45$ ). This difference may be due to the fact that they used a smoothing technique which may have slightly slowed the process by dissipating some energy. Our results are still qualitatively similar to their, however.

\subsubsection{Critical wave steepness for the onset of three-dimensional breaking waves}

The precise evaluation of $(a k)_{\text {crit }}$ is a difficult task, and one has to check that the possible numerical blow-up is due to an effective wave breaking. In order to be sure that the breaking of the wave is not due to a lack of accuracy we have to check that the simulation is grid independent. Computations are hence performed for two different grid resolutions as illustrated by an example leading to breaking of the waves when the horseshoe patterns are fully developed. Figure 7 displays the excited modes in the $\left(k_{x}, k_{y}\right)$-plane for an initial condition corresponding to a Stokes wave of steepness $(a k)_{0}=0.20$, perturbed by an unstable perturbation of class II with dominant wavevectors $\boldsymbol{k}_{1}=\left(\frac{3}{2}, 1.53\right)$ and $\boldsymbol{k}_{1}^{*}=\left(\frac{3}{2},-1.53\right)$. While for both numerical experiments eight modes are resolved in the $x$-direction (discretized over 64 collocation points), in the transverse direction either 16 or 20 modes of the perturbation are resolved (discretized over 64 and 80 collocation points respectively). Components whose energy is larger than $10^{-6}$ are depicted as a circle. For this given threshold the two grids provide the same excited modes. 

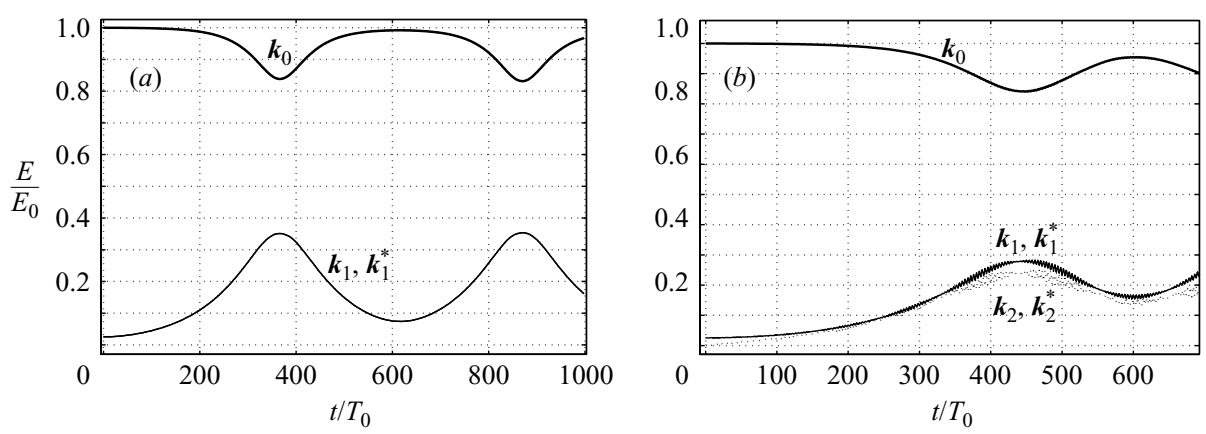

FIGURE 8. Normalized energy evolution of the fundamental mode and satellites. $(a) \boldsymbol{k}_{0}=(1,0)$, $\boldsymbol{k}_{1}=\left(\frac{3}{2}, q\right)$ and $\boldsymbol{k}_{1}^{*}=\left(\frac{3}{2},-q\right)$ with $(a k)_{0}=0.13, q=1.621, \epsilon=0.05 .(b) \boldsymbol{k}_{0}=(1,0), \boldsymbol{k}_{1}=(1, q)$, $\boldsymbol{k}_{1}^{*}=(1,-q), \boldsymbol{k}_{2}=(2, q)$ and $\boldsymbol{k}_{2}^{*}=(2,-q)$ with $(a k)_{0}=0.13, q=1.5975, \epsilon=0.05$.

We hence find that the nonlinear dynamics of the most unstable class II perturbation leads to two different regimes. For $(a k)_{0}<0.17$ the evolution is characterized by recurrence cycles (figure 8), while for greater values of initial steepness, the evolution leads to breaking.

In order to check that and to have a reasonable idea of the maximum steepness leading to a recurrence cycle, we performed a series of test with steepnesses $a k=0.16,0.17$ and 0.18 on two different resolutions where the initial perturbation is set at the most unstable class II mode in each case (taking $\epsilon=0.05$ ). We resolve either the first 7 or 15 first harmonics of the Stokes wave in the propagation direction. The 16 and 32 first harmonics of the perturbation are resolved in the transverse direction, respectively. The results show clear breaking for both simulations with $a k=0.18$, at the same time (after 120 periods of propagation), with the same energy distribution at the breaking point. Similarily, for $a k=0.16$, the simulation gives identical results and leads to recurrence cycles (see $\$ 3.2 .3$ below). The case for $a k=0.17$ is more difficult to interpret since the 'low' resolution does not lead to breaking (we limited the resolution to one cycle of recurrence when no breaking was observed, assuming that breaking would occur at the point of maximum modulation) while the 'high' resolution leads to breaking at the point of maximum modulation. In consequence we consider this value $a k=0.17$ as being a qualitatively good enough represention of the limit of breaking.

It is difficult, however, with the present method to give a more precise value than the one we have obtained. Further work could be done in this direction, by using for example a Lagrangian description which would be more appropriated to study the problem of breaking by overturning and the formation of spilling breakers.

\subsubsection{Evolution leading to recurrence}

Experiments of Su (1982) and Melville (1982) showed that the generation and amplification of crescent patterns lead to wave breaking. These experiments have been conducted for wave fields in the steepness range where the most unstable perturbation belongs to class II. We now investigate numerically the three-dimensional evolution of non-breaking waves by considering an initial wave steepness $(a k)_{0}=0.13$. We consider an initial perturbation corresponding to the most unstable mode of the class II instability with $p=\frac{1}{2}$ and $q$ as a function of $(a k)_{0}$, i.e. $q=q\left((a k)_{0}\right)$. For such steepness class II is no longer the most unstable class and the dominant instability is of class I of modulational type. A way to damp or cancel the development of 

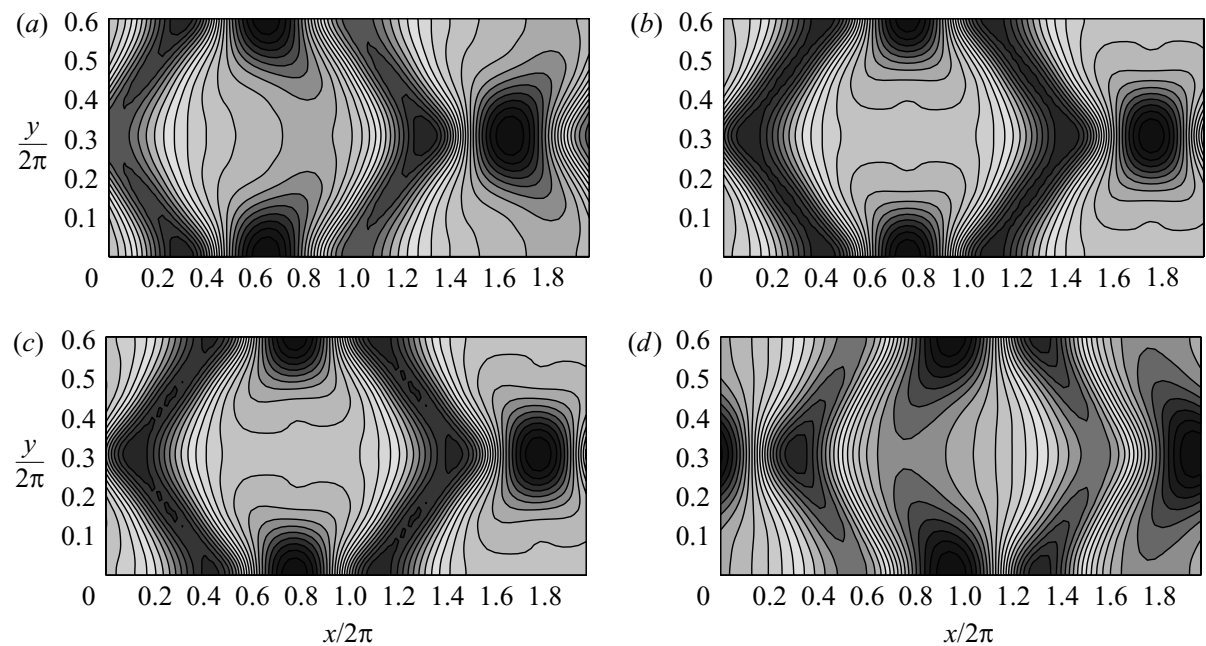

Figure 9. Iso-elevation plots corresponding to figure $8(a)$ at time $(a) t / T_{0}=340$, (b) $t / T_{0}=368,(c) t / T_{0}=372$ and $(d) t / T_{0}=420$.

the modulational instability is to consider a plastic film as was the case in the tank experiments of Collard \& Caulliez. Numerically the modulational instability can be cancelled, either by choosing $N$ such that one cannot capture unstable class I perturbations or by forcing those unstable perturbations to vanish. This means that one can artificially impose zero amplitude to unstable class I modes, hence preventing modulational instability from developing.

We find that if the initial steepness is lower than $(a k)_{\text {crit }} \simeq 0.17$, the dynamics is periodic, similar to the well-known Fermi-Pasta-Ulam recurrence phenomenon. Figure $8(a)$ shows the temporal evolution of the normalized energy of the fundamental mode of the Stokes wave and satellites corresponding to $p=\frac{1}{2}$. Iso-elevation contour plots of the surface are given in figure 9 at different instants of time corresponding to modulation and demodulation phases due to class II instability. It can be seen in figure 9 that during the phase of growth the horseshoe patterns have their front oriented forward (figure $9(a, b)$ while they have a backward orientation in the phase of decay (figure $9 c, d$ ). In the phase of growth, the number of transverse components of the spectrum extends up to eight and the front of the crescent becomes higher while the crescent itself becomes sharper as shown in figure $9(a)$. At the maximum of modulation, the top parts of the crescent tend to becomes higher and longer while during the phase of decay the spectrum narrows and the crescent separates from its centre to merge into two inverted crescents as illustrated in figure $9(d)$.

Figure 10 displays the spectral components involved in the dynamics of the wave field for two instants of time corresponding to phases of growth and decay of the crescent patterns, respectively. Eight transverse wavenumbers are needed to describe correctly the evolution and the shape of the crescent patterns. While the phase of growth is accompanied by a spectral spreading of the energy, the phase of decay corresponds to a spectral focusing as shown in figure 10 for two instants of time each side of the maximum of modulation.

Finally, figure $8(b)$ presents the same kind of evolution for a perturbation corresponding to $L_{1}$ patterns (oscillating horseshoe). The recurrence is clear and we have a similar evolution as previously described. The corresponding iso-elevation contour plots of the surface are shown in figure 11. 

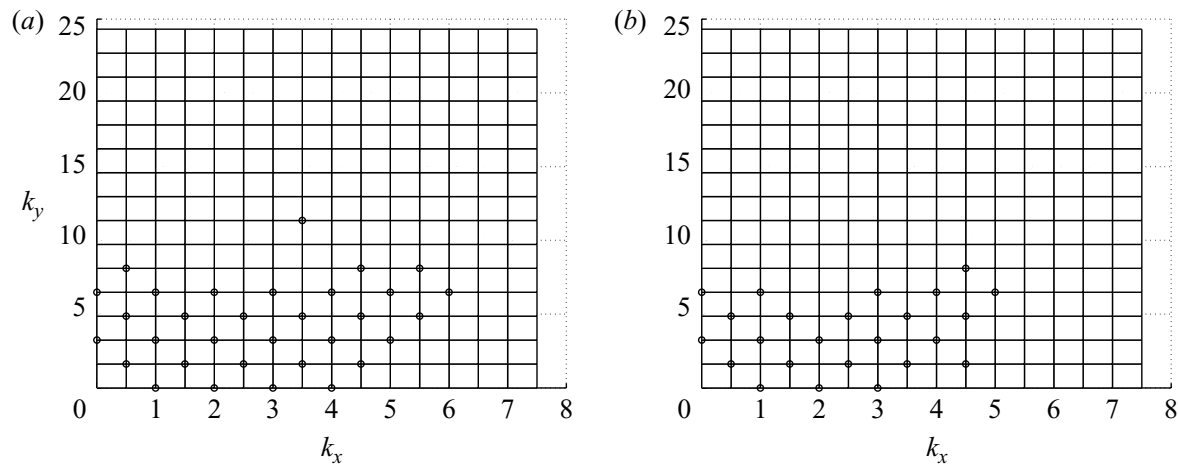

FIGURE 10. Wave-vector spectra of the wave fields corresponding to $(a)$ figure $9(a)$, (b) figure $9(d)$. The components whose energy is larger than $10^{-6}$ are depicted as a circle.
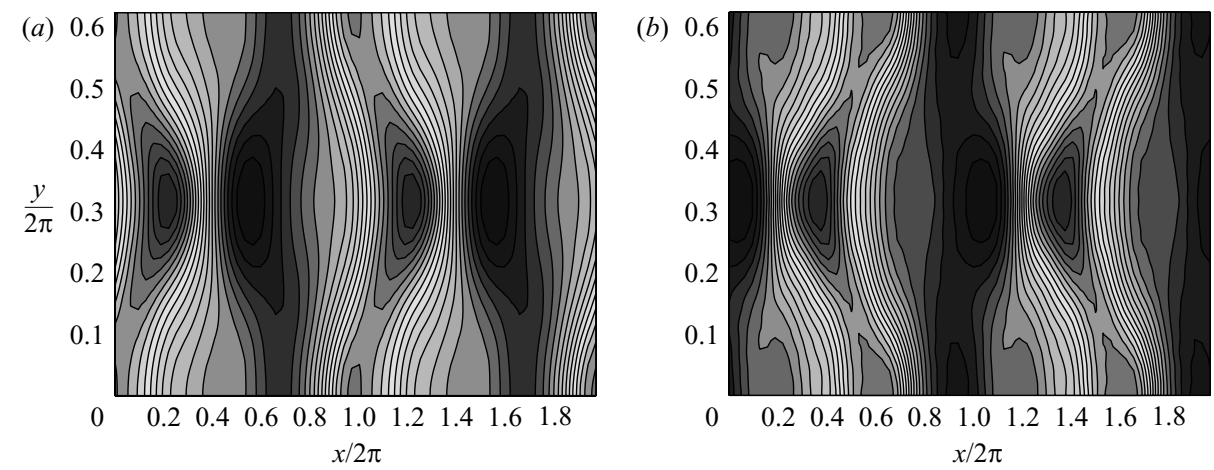

FIGURE 11. Oscillating horseshoe patterns corresponding to figure $8(b)$ at $(a) 360 T_{0}$ and $(b) 504 T_{0}$.

\section{Competition between crescent wave patterns and modulational instability}

For large initial steepness, $(a k)_{0}>0.314$, the time scale of development of class I instability is much larger than that of class II which develops quickly and evolves into a breaking wave. As mentioned previously the post-breaking dynamics is outside the scope of our numerical model. We essentially look then at the competition between instabilities of class II and between instabilities of class I and class II for $(a k)_{0}<0.314$.

We have prohibited, up to now, the development of the modulational instability by a suitable choice of the wave-tank length. This choice has been made to remove any development of this instability. For $N=2$, furthermore, it also forbids the development of class II other than $p=0$ and $p=\frac{1}{2}$. We wish here to assess the problem of competition between several class II instabilities in a first step and then to look at the competition between class I and class II instabilities for small to moderate initial steepness.

\subsection{Competition between instabilities of class II}

We investigate here the problem of the competition between several class II instabilities. We then consider a numerical wave tank with $N=4$, allowing existence and development of instabilities corresponding to $p=0, \frac{1}{4}, \frac{1}{2}$, and $\frac{3}{4}$. We keep the same grid and use as initial condition a Stokes wave with $(a k)_{0}=0.2$ plus the whole discrete spectrum of unstable perturbations. At such steepness, modulational instability would 


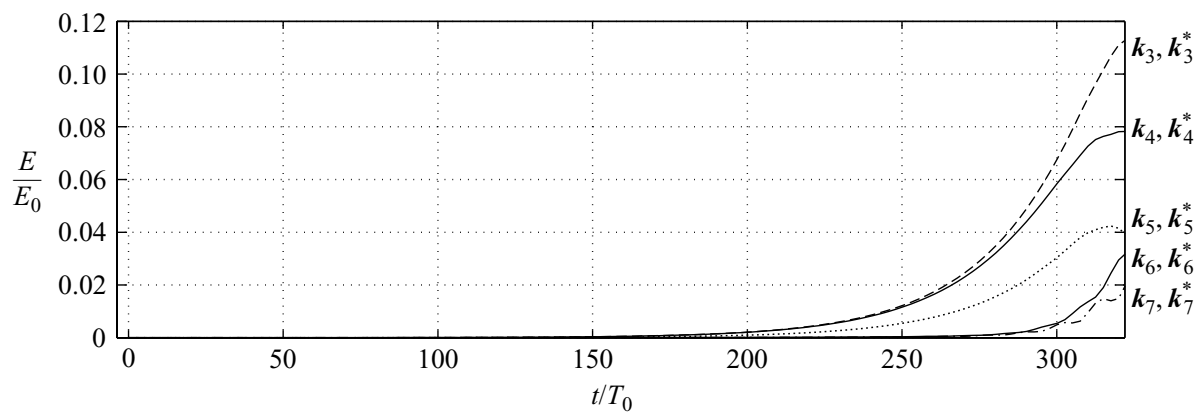

FIGURE 12. Normalized energy evolution of the dominant modes of the instabilities: $\boldsymbol{k}_{3}=\left(\frac{3}{2}, 1.53\right), \boldsymbol{k}_{3}^{*}=\left(\frac{3}{2},-1.53\right), \boldsymbol{k}_{4}=\left(\frac{5}{4}, 1.53\right), \boldsymbol{k}_{5}^{*}=\left(\frac{7}{4},-1.53\right), \boldsymbol{k}_{4} *=\left(\frac{5}{4},-1.53\right), \boldsymbol{k}_{5}=\left(\frac{7}{4}, 1.53\right)$, $\boldsymbol{k}_{6}=(1,1.53), \boldsymbol{k}_{7}^{*}=(2,-1.53), \boldsymbol{k}_{7}=(2,1.53), \boldsymbol{k}_{6}^{*}=(2,-1.53)$. The initial wave field is a Stokes wave with $(a k)_{0}=0.20$ plus the whole discrete spectrum of class II instabilities corresponding to $p=0, \frac{1}{4}, \frac{1}{2}, \frac{3}{4} . \epsilon=0.0001$.

dominate, however. We cancel these unstable perturbations of class I by restricting their amplitude to zero, preventing hence modulational instability to develop.

The result is presented in figure 12 and demonstrates that all class II instabilities may grow simultaneously. It can be noted that while the growth rate of each individual quintet is slightly smaller than the corresponding one for a single quintet, the global hierarchy due to their rate of growth is respected. Similar simulations with different initial steepness have been performed and arrive at the same results. This means that several instabilities of class II may develop and co-exist, leading to wave breaking. Competition between instabilities of class II has been also investigated by Annenkov \& Shrira (2001) within the framework of the Zakharov equation.

\subsection{Competition between instabilities of class I and class II}

It was shown that the nonlinear evolution of class I instability may lead to breaking for moderately small initial steepness (Banner \& Tian 1996, 1998). Su \& Green (1984, 1985) found experimental evidence that for moderately steep initial waves, the coupled evolution class I/class II instabilities leads to breaking.

We consider as initial conditions Stokes waves of steepnesses $(a k)_{0}=0.10,0.13$ and 0.20 respectively, disturbed by unstable perturbations corresponding to different values of $p$ and $q$. For these values of the initial steepness, it is the modulational instability which prevails.

Figure 13(a) shows the time history of the normalized energy of the fundamental mode of the Stokes wave of steepness $(a k)_{0}=0.10$ and modes $\boldsymbol{k}_{1}=\left(\frac{5}{6}, 0\right), \boldsymbol{k}_{2}=\left(\frac{7}{6}, 0\right)$, and $\boldsymbol{k}_{3}=\left(\frac{3}{2}, 1.645\right), \boldsymbol{k}_{3}{ }^{*}=\left(\frac{3}{2},-1.645\right)$. These modes are the dominant modes of class I and class II instabilities and correspond to $p=\frac{1}{6}$ and $p=\frac{1}{2}$ respectively. The class II instability generating $L_{2}$ patterns is restabilized by the modulational instability. For this value of the steepness, the transverse bandwidth of the instability region corresponding to class II is extremely narrow so that one can assume that any weak local modification of the steepness of the initial wave train will move this region and so restabilizes the class II instability corresponding to $(p, q)=\left(\frac{1}{2}, 1.645\right)$. The initial condition corresponding to figure $13(b)$ is similar to that of the previous simulation except that modulational instability is not taken into account at the start of the simulation. During the decrease of the energy of modes $\boldsymbol{k}_{3}$ and $\boldsymbol{k}_{3}^{*}$ an amplification of the dominant modes of the modulational instability is observed. For this value of $(a k)_{0}$ breaking occurs when a coupling between the two instabilities and the fundamental of 

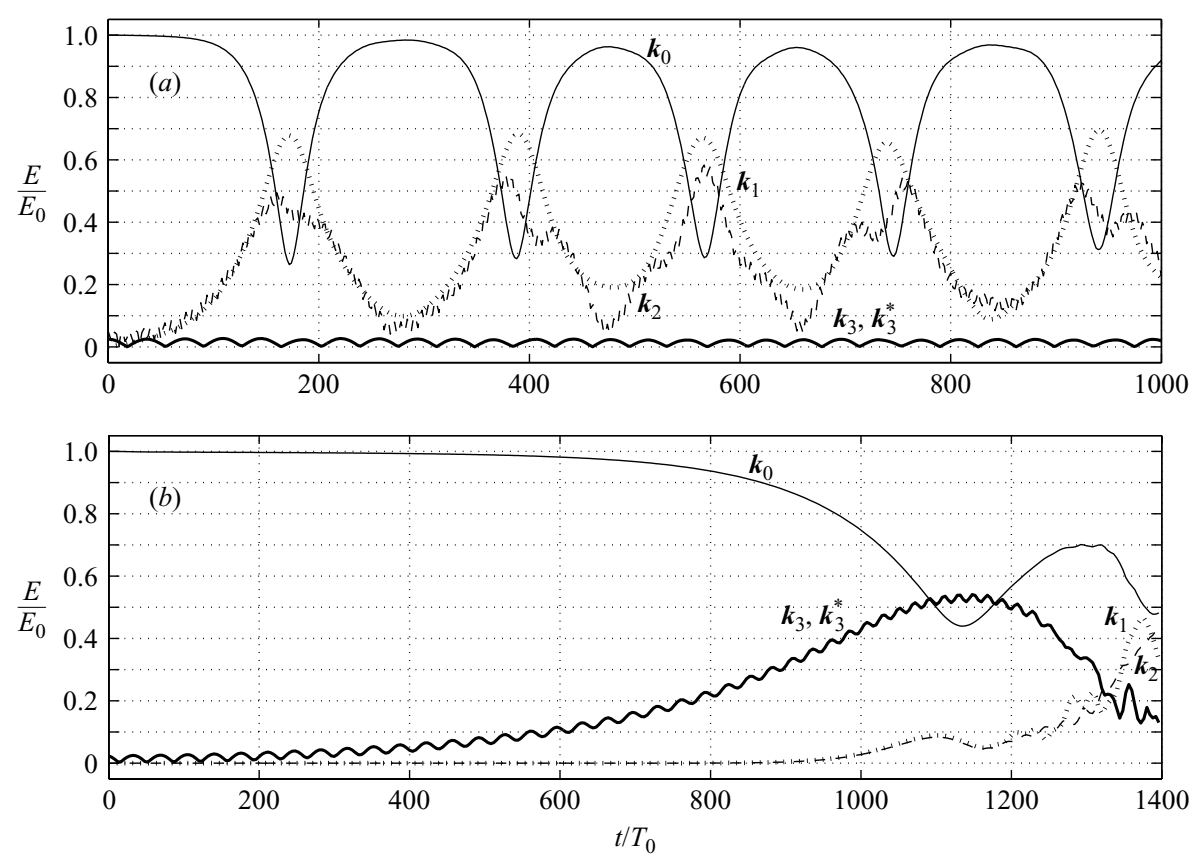

FIGURE 13. Normalized energy evolution of the main modes: $\boldsymbol{k}_{0}=(1,0), \boldsymbol{k}_{1}=\left(\frac{5}{6}, 0\right), \boldsymbol{k}_{2}=\left(\frac{7}{6}, 0\right)$, $\boldsymbol{k}_{3}=\left(\frac{3}{2}, 1.645\right), \boldsymbol{k}_{3}^{*}=\left(\frac{3}{2},-1.645\right)$. The initial wave field is a Stokes wave with $(a k)_{0}=0.10$ and the initial perturbations are: (a) $\boldsymbol{k}_{1}, \boldsymbol{k}_{2}, \boldsymbol{k}_{3}$ and $\boldsymbol{k}_{3}^{*}$ and $(b) \boldsymbol{k}_{3}$ and $\boldsymbol{k}_{3}^{*}$ only. $\epsilon=0.025$.

the Stokes wave prevails (figure 13b) while no breaking is observed in the simulation corresponding to figure $13(a)$.

Figure 14(a) corresponds to $(a k)_{0}=0.13$ and modes $\boldsymbol{k}_{1}=\left(\frac{3}{4}, 0\right), \boldsymbol{k}_{2}=\left(\frac{5}{4}, 0\right)$, and $\boldsymbol{k}_{3}=\left(\frac{3}{2}, 1.621\right), \boldsymbol{k}_{3}{ }^{*}=\left(\frac{3}{2},-1.621\right)$. These modes are the dominant modes of class I and class II instabilities and correspond to $p=\frac{1}{4}$ and $p=\frac{1}{2}$ respectively. At the maximum of modulation the growth rate of class II instability is enhanced. During the demodulation period the energy of the subharmonic and superharmonic modes of the modulational instability is decreased while the class II instability is growing, becoming dominant around $t=250 T_{0}$. For this specific value of the steepness class II instability ( $L_{2}$ pattern) is triggered by class I instability, followed by wave breaking, as suggested by $\mathrm{Su} \&$ Green $(1984,1985)$. The initial condition corresponding to figure $14(b)$ is similar to that of the previous simulation except that modulational instability is not taken into account at the beginning of the simulation. One can observe that beyond 700 fundamental periods, class I instability is excited followed by wave breaking. Here again one notices a coupling between instabilities of classes I and II and the fundamental of the Stokes wave.

For the numerical simulations corresponding to figures $15(a)$ and $15(b)$, the class II instability is now an oscillating horseshoe pattern, $L_{1}$, with dominant modes $\boldsymbol{k}_{3}=(1,1.5975), \boldsymbol{k}_{4}^{*}=(2,-1.5975)$ and $\boldsymbol{k}_{3}^{*}=(1,-1.5975), \boldsymbol{k}_{4}=(2,1.5975)$. The dominant modes of class I instability are $\boldsymbol{k}_{1}=\left(\frac{3}{4}, 0\right)$ and $\boldsymbol{k}_{2}=\left(\frac{5}{4}, 0\right)$. Figure $15(a)$ shows that the energy of the modes of the class II instability remains close to zero. At the maximum of modulation we do not observe an amplification of this instability as was the case previously for the $L_{2}$ pattern. As suggested previously, the restabilization of the perturbation corresponding to the $L_{1}$ pattern can be explained as follows. For 

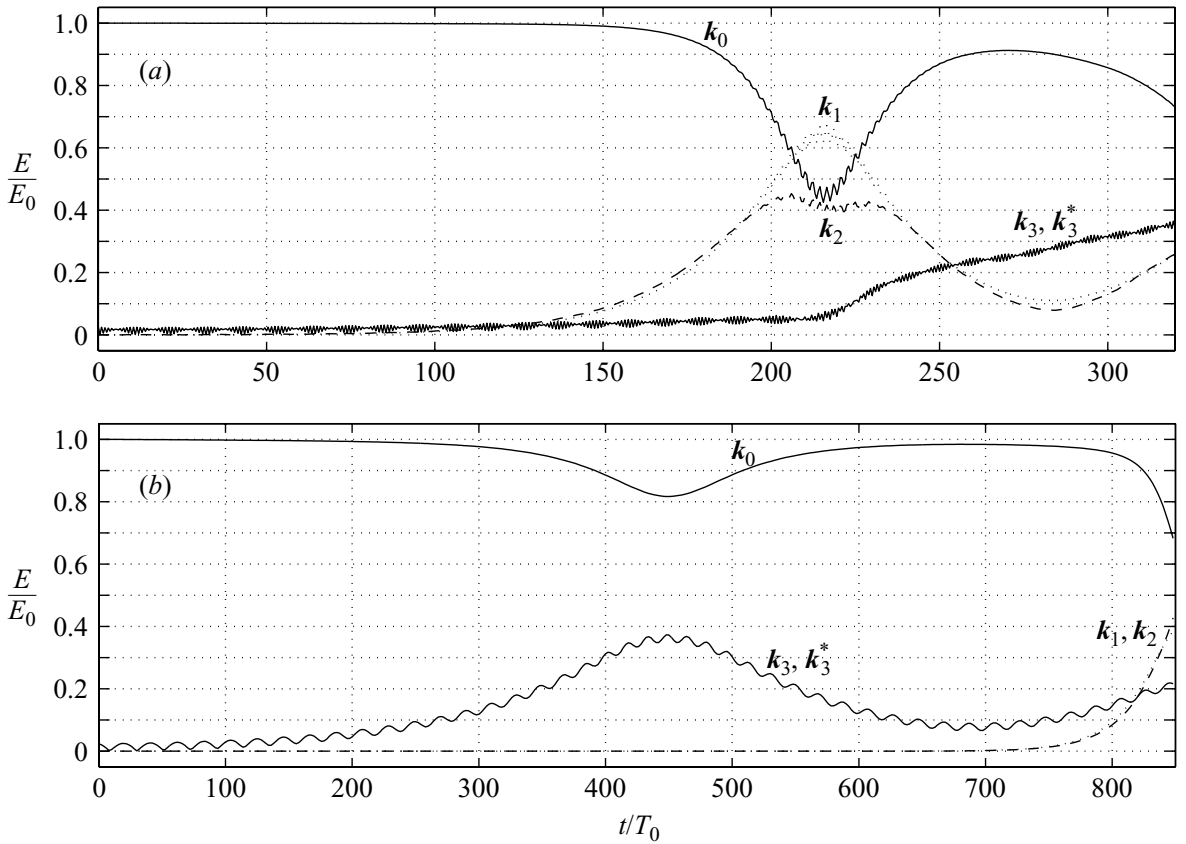

FIGURE 14. Normalized energy evolution of the main modes: $\boldsymbol{k}_{0}=(1,0), \boldsymbol{k}_{1}=\left(\frac{3}{4}, 0\right), \boldsymbol{k}_{2}=\left(\frac{5}{4}, 0\right)$, $\boldsymbol{k}_{3}=\left(\frac{3}{2}, 1.621\right), \boldsymbol{k}_{3}^{*}=\left(\frac{3}{2},-1.621\right)$. The initial wave field is a Stokes wave with $(a k)_{0}=0.13$ and the initial perturbations are: $(a) \boldsymbol{k}_{1}, \boldsymbol{k}_{2}, \boldsymbol{k}_{3}$ and $\boldsymbol{k}_{3}^{*}$ and $(b) \boldsymbol{k}_{3}$ and $\boldsymbol{k}_{3}^{*}$ only. $\epsilon=0.025$.
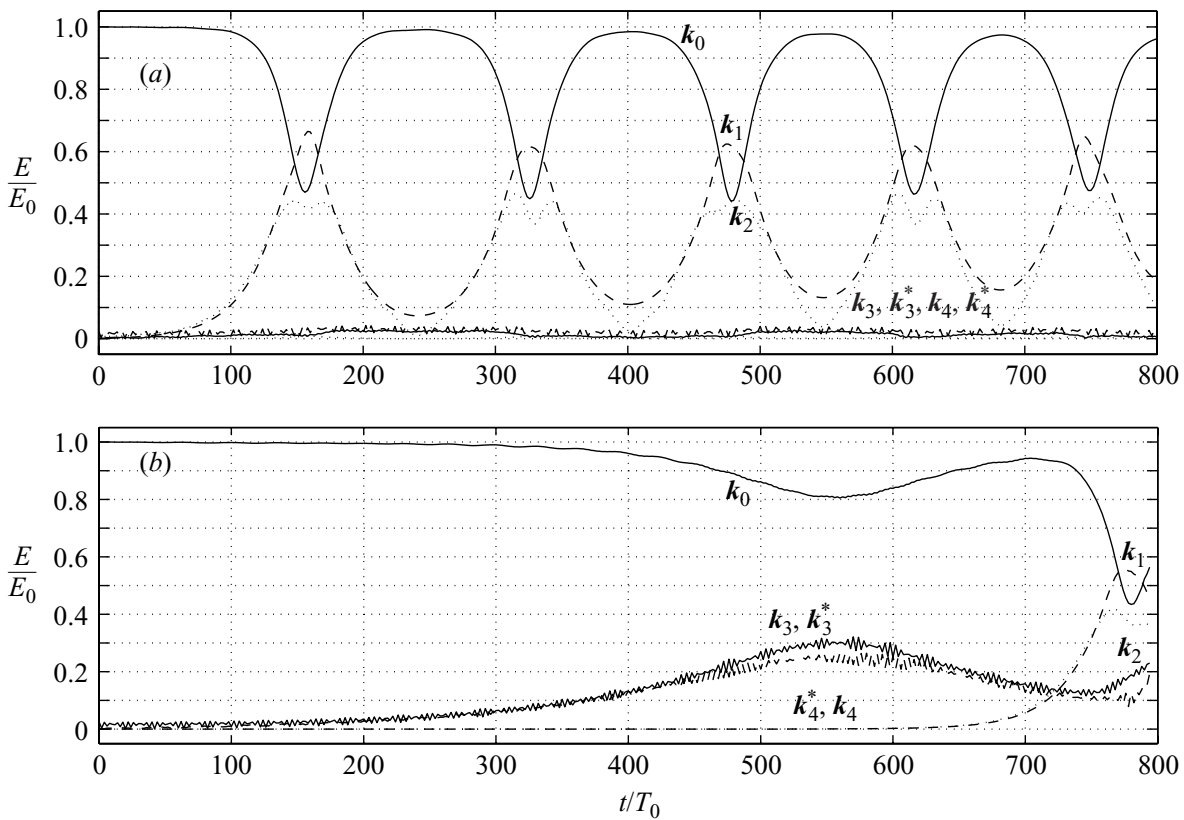

FIGURE 15. Normalized energy evolution of the main modes: $\boldsymbol{k}_{0}, \boldsymbol{k}_{1}=\left(\frac{3}{4}, 0\right), \boldsymbol{k}_{2}=\left(\frac{5}{4}, 0\right)$, $\boldsymbol{k}_{3}=(1,1.5975), \boldsymbol{k}_{4}^{*}=(2,-1.5975), \boldsymbol{k}_{3}^{*}=(1,-1.5975)$ and $\boldsymbol{k}_{4}=(2,1.5975)$. The initial wave field is a Stokes wave with $(a k)_{0}=0.13$ and the initial perturbations are: $(a) \boldsymbol{k}_{1}, \boldsymbol{k}_{2}, \boldsymbol{k}_{3}$ and $\boldsymbol{k}_{3}^{*},(b) \boldsymbol{k}_{3}$ and $\boldsymbol{k}_{3}^{*}$ only. $\epsilon=0.025$. 


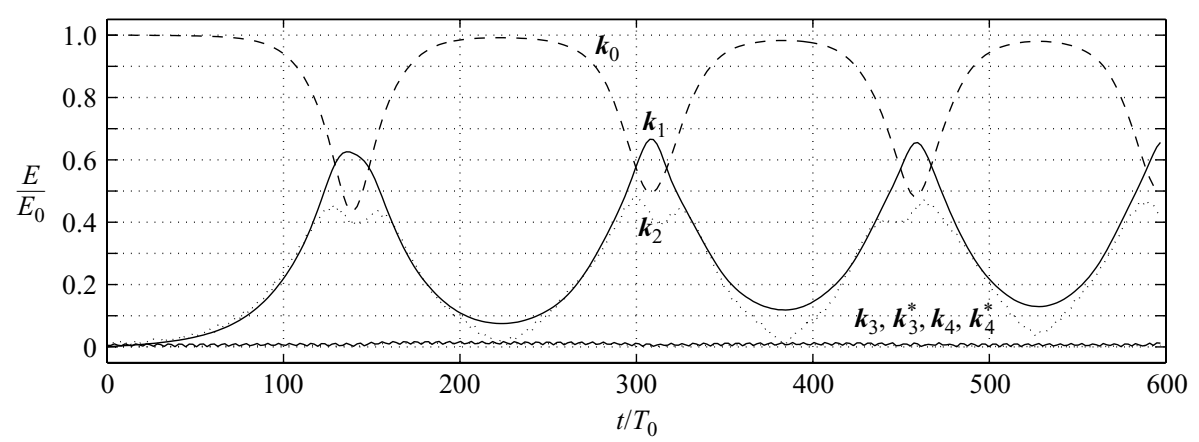

FIGURE 16. Normalized energy evolution of the main modes: $\boldsymbol{k}_{0}=(1,0), \boldsymbol{k}_{1}=\left(\frac{3}{4}, 0\right), \boldsymbol{k}_{2}=\left(\frac{5}{4}, 0\right)$, $\boldsymbol{k}_{3}=\left(\frac{3}{4}, 1.612\right), \boldsymbol{k}_{4}^{*}=\left(\frac{7}{4},-1.612\right), \boldsymbol{k}_{3}^{*}=\left(\frac{3}{4}, 1.612\right)$ and $\boldsymbol{k}_{4}=\left(\frac{7}{4}, 1.612\right)$. The initial wave field is a Stokes wave with $(a k)_{0}=0.13$ and the initial perturbations are $\boldsymbol{k}_{1}, \boldsymbol{k}_{2}, \boldsymbol{k}_{3}$ and $\boldsymbol{k}_{3}^{*} . \epsilon=0.01$.

$(a k)_{0}=0.13$ the band width of unstable transverse wavenumber is $\Delta q=0.009$ on the axis corresponding to $p=0$. The modulation of the wave train due to class I instability modifies its amplitude locally and consequently also the values of $q$ corresponding to unstable perturbations. Due to the narrowness of $\Delta q$ for $p=0$ we can expect this instability to be sensitive to the modification in steepness of the wave train and so to be restabilized. Note also that for this value of $q$ the instability corresponding to the $L_{2}$ pattern is linearly stable, explaining why this pattern is not excited during 800 fundamental periods. No breaking is observed. Figure $15(b)$ corresponds to an initial unstable perturbation due only to class II with $p=0$. Following the decrease of the modes of this instability a strong amplification of the class I modes is observed. Here, the coupled interaction of the fundamental mode of the Stokes wave with class I and class II instabilities evolves to breaking.

Figure 16 describes the case corresponding to class II instability for $p=\frac{1}{4}$ with dominant modes $\boldsymbol{k}_{3}=\left(\frac{5}{4}, 1.612\right), \boldsymbol{k}_{4}^{*}=\left(\frac{7}{4},-1.612\right)$ and $\boldsymbol{k}_{3}^{*}=\left(\frac{5}{4},-1.612\right), \boldsymbol{k}_{4}=\left(\frac{7}{4}, 1.612\right)$. The dominant modes of class I instability are $\boldsymbol{k}_{1}=\left(\frac{3}{4}, 0\right)$ and $\boldsymbol{k}_{2}=\left(\frac{5}{4}, 0\right)$. Here one observes also a restabilization of class II instability due to modulational instability. As a consequence, the $L_{4}$ pattern is not excited. No breaking is observed.

For $(a k)_{0}=0.13$, it is interesting to point out that only the most unstable pattern $L_{2}$ is triggered by the modulational instability.

Figures $17(a)$ and $17(b)$ show the time history of energy of the fundamental mode of the Stokes wave of steepness $(a k)_{0}=0.20$ and modes $\boldsymbol{k}_{1}=\left(\frac{2}{3}, 0\right), \boldsymbol{k}_{2}=\left(\frac{4}{3}, 0\right)$, and $\boldsymbol{k}_{3}=\left(\frac{3}{2}, 1.53\right), \boldsymbol{k}_{3}{ }^{*}=\left(\frac{3}{2},-1.53\right)$. These modes are the dominant modes of class I and class II instabilities and correspond to $p=\frac{1}{3}$ and $p=\frac{1}{2}$ respectively. For this value of the initial steepness the class II instability is not restabilized by the class I instability (figure 17(a). In both cases breaking occurs.

For moderate initial wave steepness $\left((a k)_{0}=0.10\right.$ and 0.13$)$, the previous simulations illustrate the importance of the interactions between class I and class II instabilities. The dominant influence of the modulational instability is emphasized when the energy level of class II instability is weak. Hence, the dominant class I restabilizes class II instabilities. When the energy of class II instability is not negligible one observes a coupling between the fundamental of the Stokes wave with both classes leading to breaking.

Our results are in agreement with the experiments of Su \& Green (1985) who showed that the coupling between class I and class II instabilities results in 

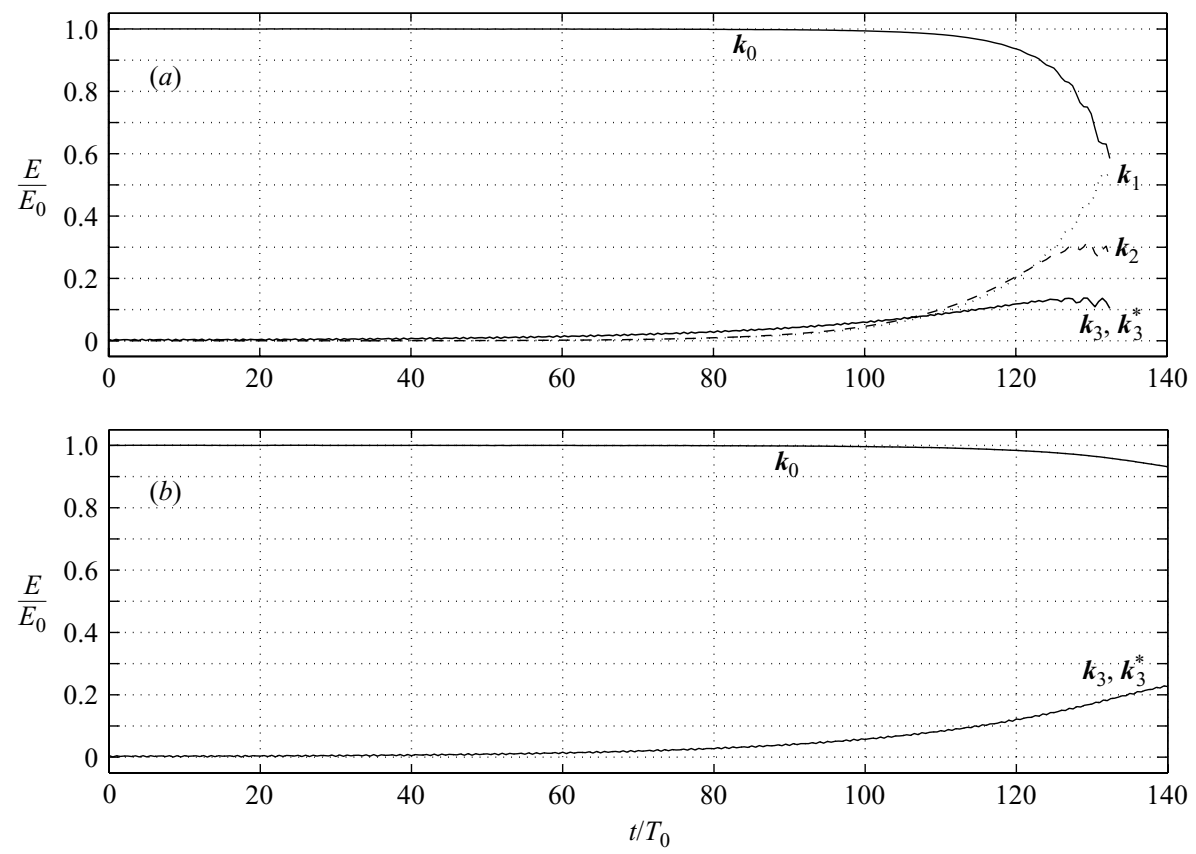

FIGURE 17. Normalized energy evolution of the main modes: $\boldsymbol{k}_{0}, \boldsymbol{k}_{1}=\left(\frac{3}{4}, 0\right), \boldsymbol{k}_{2}=\left(\frac{5}{4}, 0\right)$, $\boldsymbol{k}_{3}=\left(\frac{3}{2}, 1.53\right)$ and $\boldsymbol{k}_{3}^{*}=\left(\frac{3}{2},-1.53\right)$. The initial wave field is a Stokes wave with $(a k)_{0}=0.20$ and the initial perturbations are $(a) \boldsymbol{k}_{1}, \boldsymbol{k}_{2}$ (with $\epsilon=0.0001$ ), $\boldsymbol{k}_{3}$ and $\boldsymbol{k}_{3}^{*}$ (with $\epsilon=0.01$ ), (b) $\boldsymbol{k}_{3}$ and $\boldsymbol{k}_{3}^{*}$ only.

three-dimensional, crescent-shaped breaking waves of wave trains with the initial steepness as low as 0.12 . In their experiments class I instability grows first producing a strong modulation of the envelope followed at the maximum of modulation by the coupling between the two classes which generates three-dimensional breakers. For $(a k)_{0}>0.12$ we have observed the occurrence of a breaking due to instabilities coupling. For $(a k)_{0}=0.10$ we did not observe breaking waves if we start the numerical simulation with the initial Stokes wave perturbed by a single instability corresponding to class I, while it is not the case if we start with a single class II instability. In the second case the class I instability is excited later and the instability coupling leads to the breaking. Note that the latter case does not correspond to the Su \& Green experiments. Note that the threshold value when both class I and class II instabilities are interacting seems to be a little greater than the value obtained by Banner \& Tian for a pure two-dimensional wave field.

Furthermore, these results are in accordance with those obtained by Stiassnie \& Shemer (1987) who used the modified Zakharov equation to study the coupled evolution of class I and class II instabilities. They found a similar tendency of class I to suppress class II whenever the level of class I disturbance was substantially higher than that of class II, which is in agreement with our results.

\section{Finite depth effect on class competition}

The nonlinear evolution of class II instabilities has mostly been studied in the case of infinite depth. We are investigating their behaviour on finite depth for $k h=1$ where $h$ is the depth. A more detailed analysis will be presented in a forthcoming paper. 


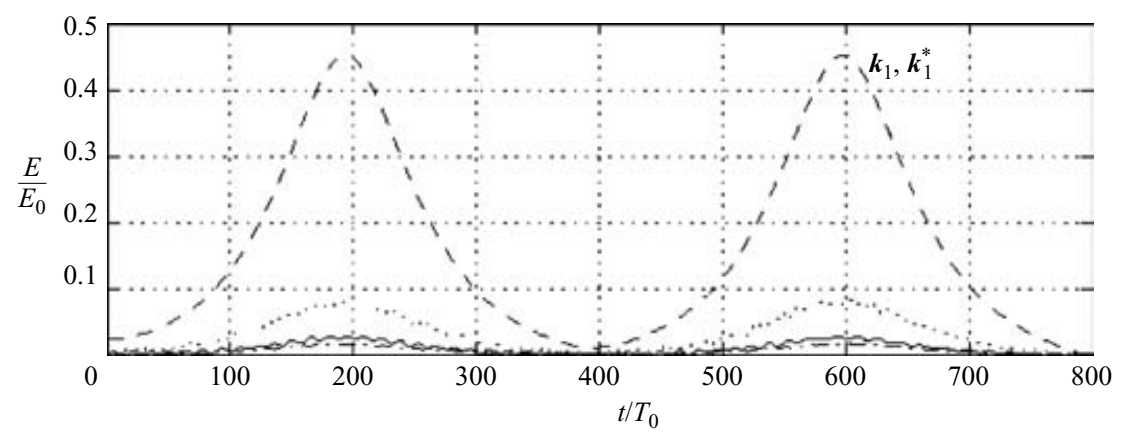

FIGURE 18. Recurrence for class II instability with $(p, q)=\left(\frac{1}{2}, 0.98\right), k h=1,(a k)_{0}=0.11$. The instability with dominant modes $\boldsymbol{k}_{1}=\left(\frac{3}{2}, 0.98\right)$ and $\boldsymbol{k}_{1}^{*}=\left(\frac{3}{2},-0.98\right)$ is triggered with $\epsilon=0.05$. The other curves represent the higher-order harmonics of the instability.

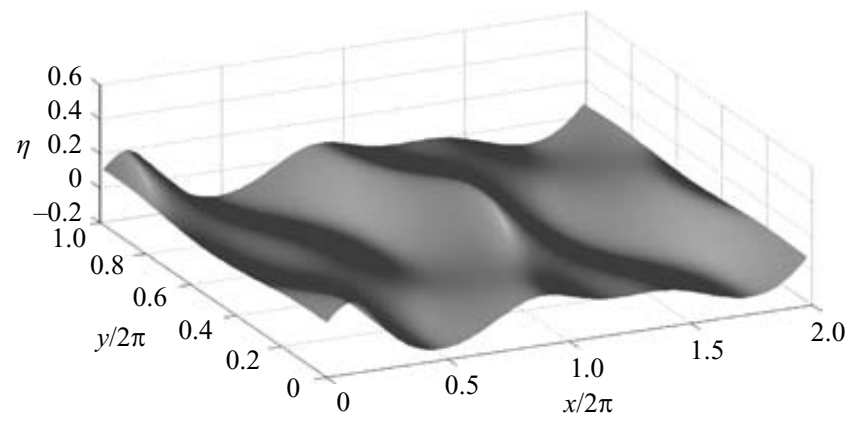

FIGURE 19. Surface elevation corresponding to figure 18 at $t / T_{0}=160$.

Here we focus our attention on the effect of the depth on the competition between classes.

On finite depth, the overall behaviour of 5-wave interactions generating the crescent patterns is similar to that corresponding to infinite depth. A series of recurrence cycles occurs for small values of wave steepness, and breaking for higher values. For $k h=1$ and $(a k)_{0}=0.11$, figure 18 shows the time history of the energy of the dominant harmonics $\boldsymbol{k}_{1}=\left(\frac{3}{2}, 0.98\right), \boldsymbol{k}_{1}^{*}=\left(\frac{3}{2},-0.98\right)$ and neighbouring harmonics of the most unstable perturbation of class II leading to the occurrence of $L_{2}$-patterns. A recurrence phenomenon is observed. A surface elevation plot is given in figure 19. The transition between recurrence and breaking is around $(a k)_{0}=0.13$ for the most unstable perturbation of class II. As for infinite depth, an oscillating $L_{1}$ horse-shoe pattern develops for $\boldsymbol{k}_{3}=(1, q), \boldsymbol{k}_{4}^{*}=(2,-q)$ and $\boldsymbol{k}_{3}^{*}=(1,-q), \boldsymbol{k}_{4}=(2, q)$. For $k h=1$ and $(a k)_{0}=0.20$, figure 20 demonstrates the co-existence of $L_{2^{-}}$and $L_{1}$-patterns corresponding to transverse wavenumber $q=0.91$.

It is well known that the class I instability band detaches from the $p$-axis when $k h<1.363$. This critical value corresponds to a restabilization of the two-dimensional long-wave perturbations. It is possible to exclude the class I instabilities by using a sufficiently narrow tank. The transverse spectral discretization must be chosen (i.e. tank width small enough) not to include the class I instability region. The longitudinal spectral discretization can be arbitrarily fine. In this case it is not necessary to invoke the presence of a plastic film to cancel or attenuate the modulational instability. 


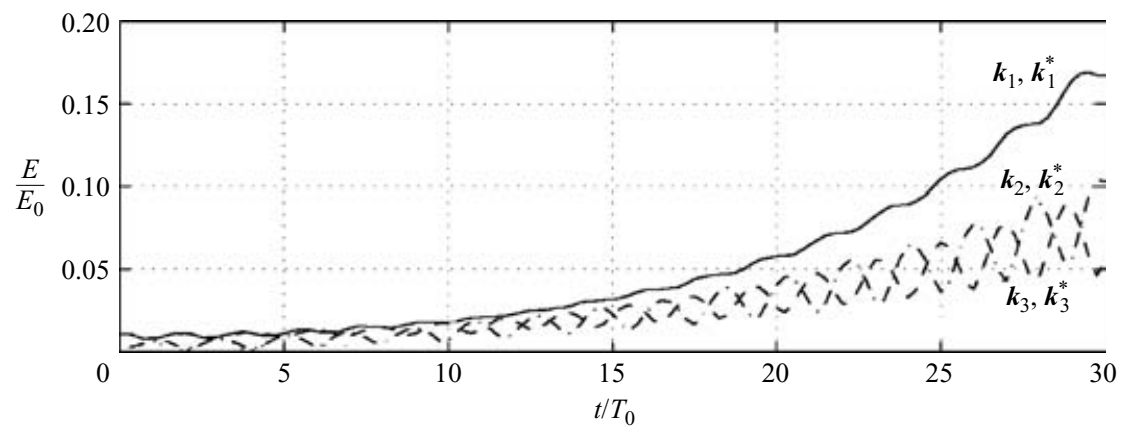

Figure 20. Coexistence of two class II instabilities for $k h=1$ and $(a k)_{0}=0.20$. The dominant modes of the instabilities corresponding to phase-locked horseshoe patterns are $\boldsymbol{k}_{1}=\left(\frac{3}{2}, 0.91\right), \boldsymbol{k}_{1}^{*}=\left(\frac{3}{2},-0.91\right)$ and those corresponding to oscillating patterns are $\boldsymbol{k}_{2}=$ $(1.0,0.91), \boldsymbol{k}_{2}^{*}=(1.0,-0.91)$ and $\boldsymbol{k}_{3}=(2,0.91) \boldsymbol{k}_{3}^{*}=(2,-0.91)$. The instabilities are triggered with $\epsilon=0.02$.

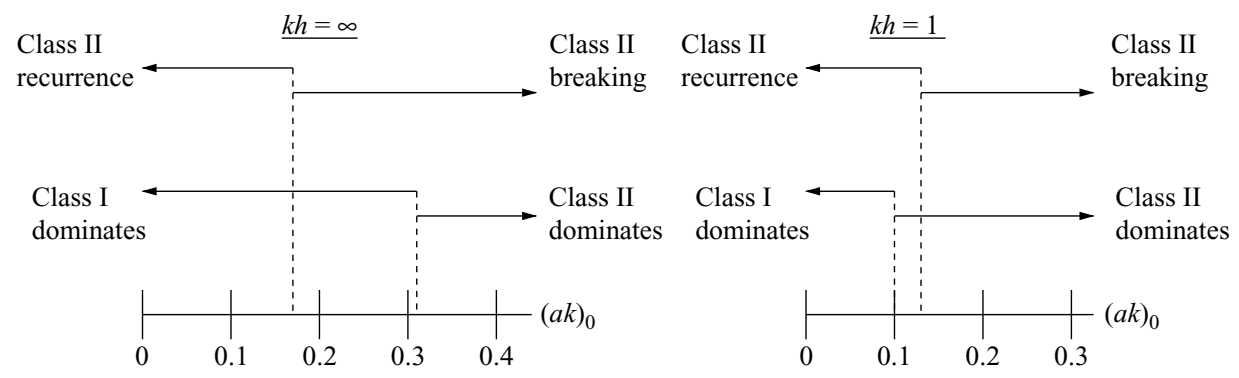

FIGURE 21. Steepness threshold for recurrence vs. breaking and predominance of class I instabilities vs. class II instabilities for $k h=\infty$ and $k h=1$.

When the depth is reduced from $k h=\infty$ to $k h=1$, the relative strength of class I and II instability changes. In general, higher-order instabilities (quintet, sextet,...) become more important for shallower water (Francius \& Kharif 2005). The growth rates of the most unstable class I quartet interaction and the most unstable class II quintet interaction (see McLean 1982b) can be used to define which is the dominating instability for a given depth and steepness. For the infinite depth case, the steepness for which the maximal growth rates of class I and class II are equal is $(a k)_{0}=0.314$, whereas for $k h=1$, the threshold steepness is only 0.10 . Note that the highest values of the steepness of a Stokes wave train are 0.4432 and 0.325 for $k h=\infty$ and $k h=1$ respectively. Furthermore, class I instability seems not to cancel class II instability unless its growth rate is significantly larger than that of class II. For infinite depth class II instabilities are dominant for strongly nonlinear Stokes waves while for $k h=1$ they are dominant for moderately nonlinear water waves. We can conclude that it should be easier to observe crescent wave patterns in finite depth rather than in infinite depth. Figure 21 shows the critical values of the steepness separating class I and class II predominance. For a wave steepness larger or smaller than this critical value it is instabilities of class II or class I which dominate, respectively. In both cases, the threshold steepness separating recurrence and breaking phenomena for class II instabilities is given. It is seen that for $k h=1$, class II instability dominates for all of the range where breaking occurs. 

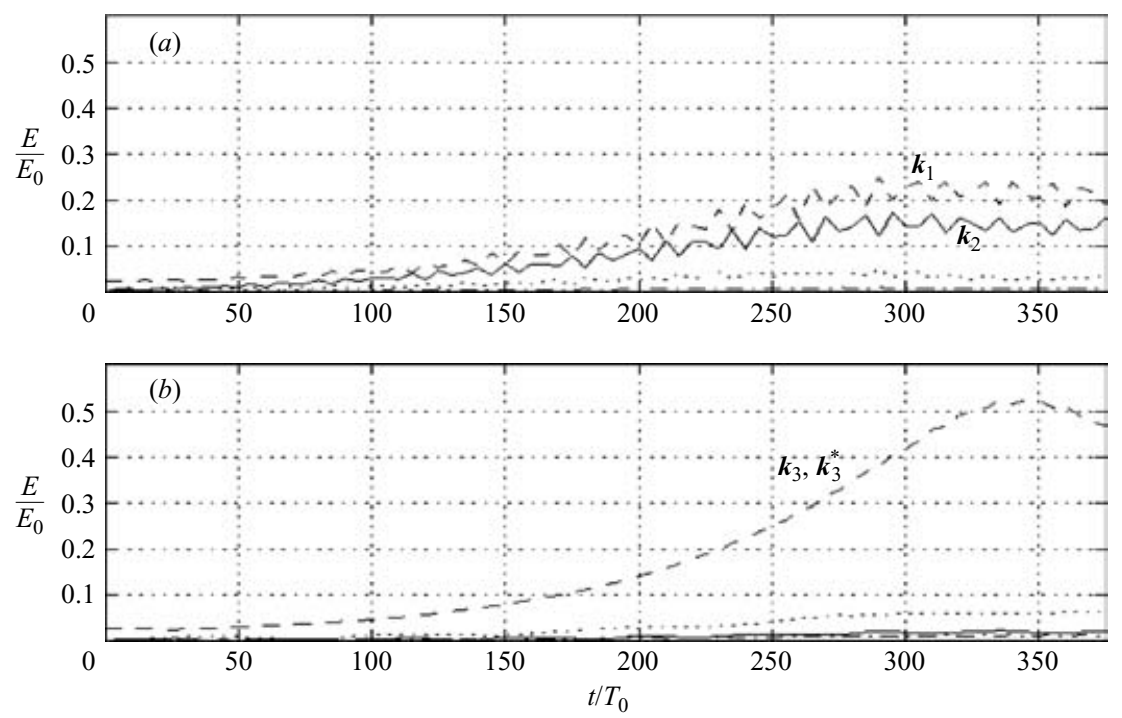

FIGURE 22. Coexistence of class I and class II instabilities for $k h=1$ and $(a k)_{0}=0.10$. (a) development of the dominant modes $\boldsymbol{k}_{1}=\left(\frac{3}{2}, 0.31\right)$ and $\boldsymbol{k}_{2}=\left(\frac{1}{2}, 0.31\right)$ of the unstable class I perturbation for $(p, q)=\left(\frac{1}{2}, 0.31\right)$. (b) Development of the dominant modes $\boldsymbol{k}_{3}=\left(\frac{3}{2}, 0.992\right)$ and $\boldsymbol{k}_{3}^{*}=\left(\frac{3}{2},-0.992\right)$ of the unstable class II perturbation for $(p, q)=\left(\frac{1}{2}, 0.992\right)$. The instabilities are triggered with $\epsilon=0.05$.

For $k h=1$, it is expected that class I and class II should coexist for realistic values of the steepness, namely $(a k)_{0}=0.10$, if the chosen unstable perturbations have similar growth rates, even in a wide tank. This is indeed what is observed in a simulation where $\boldsymbol{k}_{1}=\left(\frac{3}{2}, 0.31\right), \boldsymbol{k}_{2}=\left(\frac{1}{2},-0.31\right)$ (class I) and $\boldsymbol{k}_{3}=\left(\frac{3}{2}, 0.992\right), \boldsymbol{k}_{3}^{*}=\left(\frac{3}{2},-0.992\right)$ (class II) both are triggered with $\epsilon=0.05$ (see figure 22 ). The growth rate evolutions are very similar for the first $\simeq 150$ periods, but the class II instability then grows larger than the class I instability.

\section{Observability of oscillating crescent patterns in water wave tanks}

We now focus our attention on the problem of the observation of $L_{1}$ patterns in water wave tanks. While the particular case of $L_{2}$ patterns due to the growth of the most unstable perturbation of class II is well understood, the mechanism responsible of the generation of $L_{1}$ patterns is less understood. For sufficiently steep Stokes waves the dominant instability is that corresponding to $L_{2}$ patterns and it is natural to observe these structures which evolve into three-dimensional spilling breakers. Collard \& Caulliez (1999) reported observation of $L_{1}$ patterns, however. They emphasized that the selection mechanism of such patterns was still unexplained. For moderately steep Stokes waves propagating on the free surface of an unbounded fluid, the most unstable perturbation is of class I and the dominant instability of class II corresponds to $L_{2}$ patterns. It is surprising to observe $L_{1}$ patterns. Due to finite tank width these patterns are excited by noise or parametric resonance as shown below. From this point of view instabilities corresponding to $L_{1}$ patterns can become the dominant ones in confined geometries. Our goal in this section is to suggest another possible mechanism for the generation of the oscillating structures in water wave tanks. The parametric instabilities due to the wave-maker can promote and force linearly unstable perturbations of Stokes wave trains. 

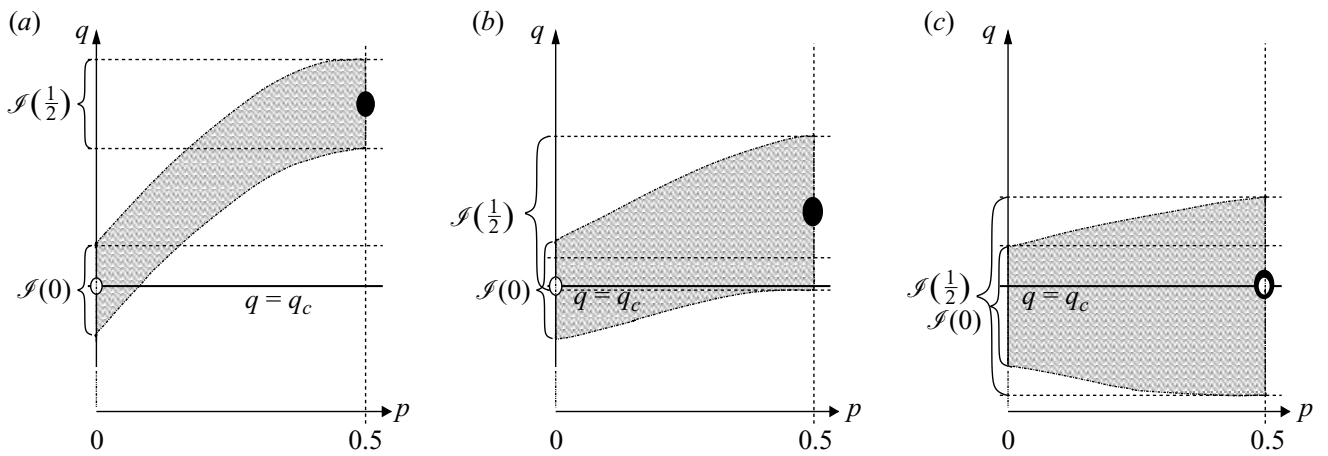

FIGURE 23. Schematic instability diagrams of class II focusing on the unstable region with $0 \leqslant p \leqslant 0.5$. The different cases depict the three regimes in infinite depth corresponding typically to $(a)(a k)_{0}<0.17,(b) 0.17<(a k)_{0}<0.2$ and $(c)(a k)_{0}>0.2$. The black dot labels the most unstable perturbation of class II and the white dot labels the most unstable mode along the line $q=q_{c}$.

\subsection{Conditions for the occurrence of oscillating crescent patterns}

From the previous results, we now present the conditions required for the appearance of $L_{1}$ patterns. To generate such structures in water wave tanks, the following conditions have to be fulfilled:

(i) An attenuation of modulational instability

Since we have to consider a moderately steep initial wave and since we have seen that in such a context class I is dominant, in most cases cancelling any further development of class II instability, class I has to be attenuated or prohibited. This can be achieved in wave facilities by putting a plastic film on top of the fluid (Collard \& Caulliez 1999). Note that in finite depth (for $k h<1.363$ ) the strength of the dominant instabilities of class I and class II are of same order when the wave is moderately steep. Thus, in this case, the class I instability is no longer the predominant one.

(ii) A selection mechanism of an unstable transverse wavenumber

This means that through a series of specific mechanisms, depicted in $\S 6.2$, a transverse wavenumber is selected. Let us denote by $q_{c}$ this transverse wavenumber which falls in the class II instability domain of the $(p, q)$-plane. However, note that an alternative selection mechanism described by Furhman et al. (2004) can prevail to explain the occurrence of $L_{1}$ patterns.

(iii) A weakly nonlinear initial wave train for the selected transverse wavenumber

It is well known that the most unstable perturbation of class II is for $p=\frac{1}{2}$, corresponding to $L_{2}$ patterns. We have seen moreover that several class II instabilities may coexist and develop together. To obtain $L_{1}$ patterns one has thus to make sure that the perturbation corresponding to $(p, q)=\left(0, q_{c}\right)$ is the most unstable one along the straight line $q=q_{c}$ in the $(p, q)$-plane. In order to satisfy this condition, the projection onto the $q$ axis of a neighbourhood of the most unstable perturbation of class II $\left(p=\frac{1}{2}\right)$ must not include $q_{c}$. Figure 23(a) illustrates such a condition where $\mathscr{I}(p)$ is the projection on the $q$-axis of the intersection of the domain of instability with the straight line $p=1 / n$. Here the bands $\mathscr{I}(0)$ and $\mathscr{I}\left(\frac{1}{2}\right)$ are disconnected, and if $q_{c}$ is included in $\mathscr{I}(0)$, the mode $\left(0, q_{c}\right)$ may be the most unstable one along the straight line $q=q_{c}$. This condition can only be satisfied for small values of $(a k)_{0}$, typically for $(a k)_{0}<0.17$ (in deep water). A steepening of the initial wave field corresponds to a flattening of the domain of instability of class II and a broadening 


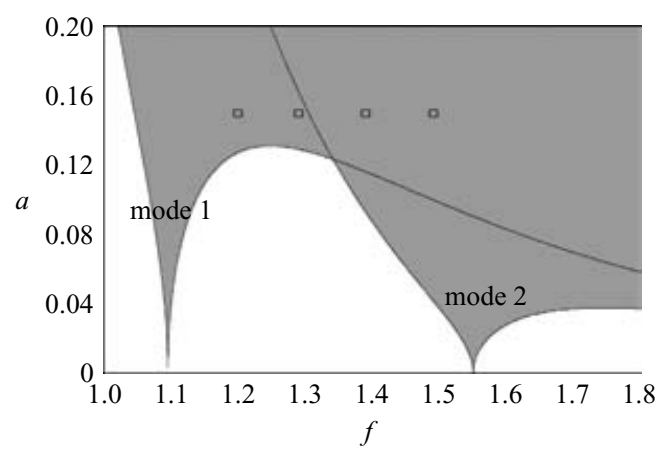

FIGURE 24. Margin of stability of the two first cross-wave modes. The solid lines are neutral wavemaker amplitude as a function of wavemaker frequency while the grey areas represent unstable regions and correspond to a growth of the cross-waves. The wavemaker parameters correspond to the experimental setup by Collard \& Caulliez while the squares indicate the location of some of their experiments.

along its axis of symmetry. When $(a k)_{0}$ increases, the two regions $\mathscr{I}(0)$ and $\mathscr{I}\left(\frac{1}{2}\right)$ may overlap, but the previous condition can still be fulfilled in a transition regime as shown in figure $23(b)$. For $(a k)_{0}>0.2$, the band $\mathscr{I}(0)$ is now included in $\mathscr{I}\left(\frac{1}{2}\right)$ due to the flattening of the unstable region. Figure 23(c) illustrates this regime where the most unstable perturbation along the straight line $q=q_{c}$ is no longer on the $q$-axis, whatever arbitrary choice of $q_{c}$ is made. The dominant instability corresponds now to $p=\frac{1}{2}$ and $L_{2}$ patterns should occur.

These three conditions appear then as requirements for the development of oscillating patterns.

\subsection{Selection mechanism of the unstable transverse wavenumber}

This selection involves two main steps.

\subsubsection{Parametric resonance of cross-waves}

Since Faraday's first experiments, it has been well known that parametrically excited cross-waves may be generated in a tank by the motion of the wavemaker (Garrett 1970; Mahony 1972; Jones 1984; Miles 1988; Miles \& Becker 1988). If the frequency of excitation (that of the wavemaker) approximates twice one of the resonant frequencies of the transverse standing wave modes and if the amplitude of excitation exceeds a threshold, energy is transferred from the wavemaker motion to the cross-waves through nonlinear interaction.

This transfer is possible if the half-frequency of excitation $\omega / 2$ approximates one of the natural frequencies

$$
\omega_{n}=\sqrt{n g \pi / b} \quad(n=1,2, \ldots)
$$

where $b$ is the width of the tank and where the tank is assumed of infinite depth (in fact the value of the dispersive parameter $k h$ for the first transverse eigenmode is greater than 1, suggesting that finite depth effects do not drastically change the frequency and the domain of instability of this eigenmode). In this case Jones (1984) obtained the evolution equations which govern the growth of these cross-waves. We can hence derive, for a given experimental setup, the margin of stability of each of these crosswave modes which gives us the minimum wavemaker amplitude able to excite those cross-waves. Figure 24 displays the margin of stability computed from Jones's results 
(corrected by considering the sign errors in Jones's equations (41)-(42) as mentioned by Lichter \& Chen 1987) for the two first cross-wave modes corresponding to Collard \& Caulliez's experiments. In their experiments the wavemaker is of the flap type and the maximum displacement $a$ rose to $15 \mathrm{~cm}$ when the wavemaker frequency was in the range $1.2 \mathrm{~s}^{-1}<f<1.5 \mathrm{~s}^{-1}$. This made possible excitation of the two first cross-wave modes in this case.

\subsubsection{Superharmonic cascade}

Once the first or the first two transverse eigenmodes of the channel are excited, they nonlinearly interact with the basic Stokes wave train. This leads to excitation of higher components and a transfer of energy to higher transverse wave modes of the channel.

As an example of such nonlinear interactions, we consider a simulation with initial wave steepness $(a k)_{0}=0.17$. For such a steepness, the class II modes which are involved in the quintet interactions corresponding to the development of the $L_{1}$ pattern are $\boldsymbol{k}_{0}=(1,0), \boldsymbol{k}_{4}=\left(1, q_{c}\right), \boldsymbol{k}_{5}^{*}=\left(2,-q_{c}\right), \boldsymbol{k}_{4}^{*}=\left(1,-q_{c}\right)$ and $\boldsymbol{k}_{5}=\left(2, q_{c}\right)$. The maximum growth of these instabilities corresponds to $q_{c}=1.551$. In a wave tank of width $b$, the smallest transverse wave-vector component is $k_{\perp}=\pi / b$ and higher harmonics are $n k_{\perp}$. If there exists an integer $n$ which satisfies $n k_{\perp}=q_{c}$ one can expect the class II instability to be excited. Herein we choose $n=8$. This means that $q_{c}=1.551$ corresponds to the eighth transverse eigenmode of our numerical channel. We also check that no other transverse eigenmode lies in the class II instability region. For illustration, the initial perturbation is hence chosen to be set to the first transverse eigenmode of the channel so as to simulate the first cross-wave which may be parametrically excited. As its growth rate is close to that of the class I instability, the relative amplitude of the perturbation is taken as $\epsilon=0.1$. Figure 25 presents the results of such a simulation. It displays the evolution of the normalized energy of the fundamental of the Stokes wave and the most amplified modes. Here the energy is transferred from the first eigenmode of the channel to higher transverse harmonics by nonlinear interaction with the Stokes wave. When energy is increased to the eighth transverse eigenmode, the instabilities involving $\boldsymbol{k}_{0}, \boldsymbol{k}_{4}, \boldsymbol{k}_{5}^{*}$ and $\boldsymbol{k}_{0}, \boldsymbol{k}_{4}^{*}, \boldsymbol{k}_{5}$ start to grow. This transfer is further illustrated in figure $25(c, d)$ where a wave-vector spectrum description of the modes involved at several instants of time of the simulation is displayed. Finally, figure 25(b) shows a surface elevation plot at the end of the simulation corresponding to $L_{1}$ patterns (to be compared with Collard \& Caulliez (1999) figure $1 b$ ). We performed a numerical simulation where the initial condition includes the second cross-wave mode instead of the first mode. The oscillating structures are now observed to grow with a characteristic time scale decreased by factor two. A further simulation with the two first cross-wave modes gave the same result: the time scale of $L_{1}$ patterns is reduced by a factor two. When the second cross-wave mode is taken into account the characteristic time scale for the occurrence of the aligned crescent structures is close to $370 T_{0}$ (half of the period given by figure 25). The linear stability analysis tells us that the characteristic time scale of class II instability is of $O\left((a k)_{0}^{-3} T_{0}\right)$. Hence for $(a k)_{0}=0.17$, this time scale is close to $200 T_{0}$. This means that the time scale of the occurrence of the oscillating patterns is multiplied by about one and a half because of the superharmonic cascade. This factor can be significantly reduced if the initial amplitude of the cross-wave modes is increased. The quantitative difference observed between the experimental and numerical characteristic time scales is due to the presence of a plastic film in the experiments. The experiment for $(a k)_{0}=0.17$, with the plastic film, does not correspond to a Stokes wave with this wave steepness. 
(a)

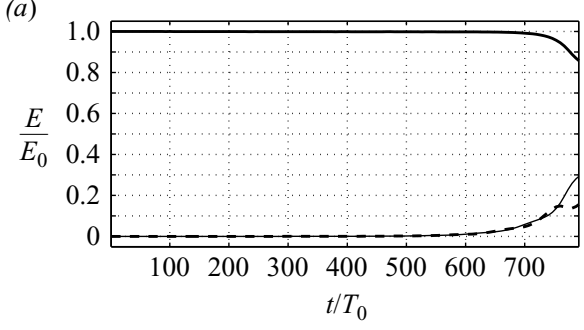

(c)

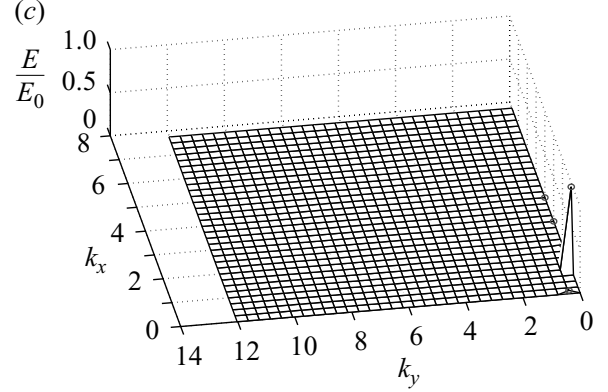

(b)

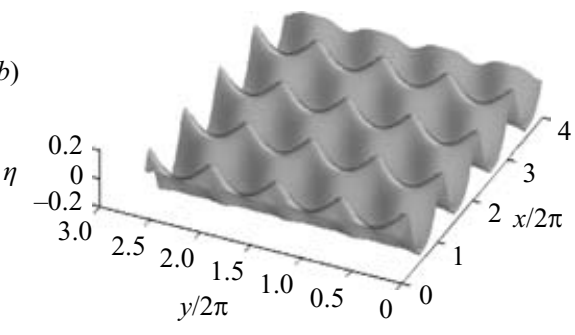

(d)

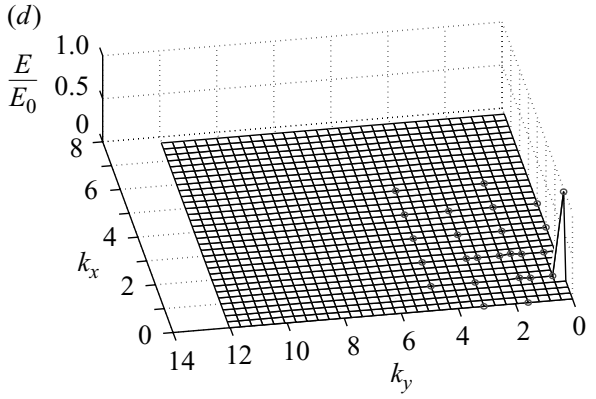

FIgURE 25. Evolution of a perturbed Stokes wave train of initial steepness $(a k)_{0}=0.17$ and $\epsilon=0.10$ (the wave-vector spectrum of the initial condition is given in $(c)$ ). (a) Normalized energy evolution of the main modes $\boldsymbol{k}_{0}=(1,0)$ (thick solid line), $\left(1, \pm q_{c}\right)$ (thin solid line) and $\left(2, \mp q_{c}\right)$ (dashed line) with $q_{c}=1.58$. (b) Free-surface elevation at $t / T_{0}=740 .(c, d)$ wave-vector spectra of the free surface at $t / T_{0}=0$ and $t / T_{0}=740$. Harmonics with energy larger than $10^{-6} E_{0}$ are depicted as a circle.

As shown by Furhman et al. (2004) the experiments correspond to higher values of the wave steepness. This explains why the period of occurrence of these structures is strongly reduced.

It can be added here that, in this example, the numerical grid was chosen to allow modulational instability to develop. In the case where only the first cross-waves were excited at the beginning of the simulation, we observed no development of class I instability (as depicted in figure 25). If modulational instability and cross-waves are both included in the initial condition, class I develops, leading to breaking, however. The growth of the modulational instability avoids the development of the oscillating patterns. This explains why it is necessary to attenuate the growth of the modulational instability to observe these patterns.

The competition between the two generating processes deserves much more study. This is not our aim here and we just want to show that parametric excitation may play a major role in the whole process by speeding up the development of $L_{1}$ patterns.

While we have shown that parametric resonance, in a wave tank, may promote transfer of energy to the first cross-wave modes, nonlinear interaction with the basic Stokes wave leads to a rise of higher transverse eigenmodes of the channel. Those eigenmodes are then amplified and a low level of energy is sufficient to trigger instabilities of class II whose transverse wavenumber corresponds to these excited transverse eigenmodes.

\subsection{Numerical simulation}

The mechanisms described in the previous subsection are relevant to explain the occurrence of the $L_{1}$ pattern observed by Collard \& Caulliez (1999). Following their 


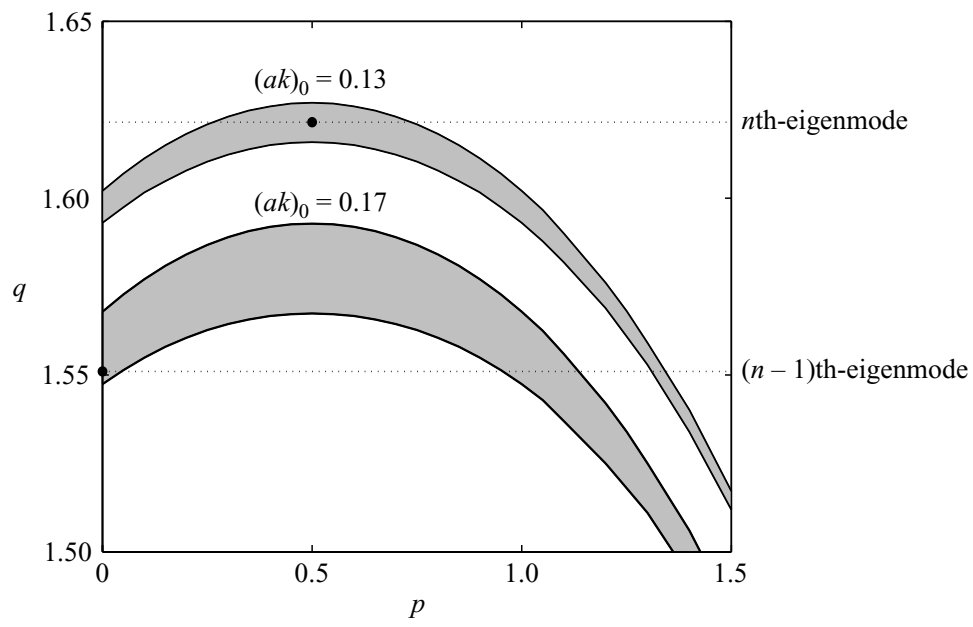

FIGURE 26. Class II instability diagrams for wave steepness $(a k)_{0}=0.13$ and $(a k)_{0}=0.17$. The horizontal lines correspond to two consecutive eigenmodes of the numerical channel considered while the dots represent the most unstable perturbation along those horizontal lines.

experiments, we consider two different values of the wave steepness, in the range $0.13<(a k)_{0}<0.17$. In order to reproduce the experimental results, we must observe emergence of $L_{2}$ patterns for the steepness $(a k)_{0}=0.13$ and $L_{1}$ patterns for a higher steepness. This means that if the considered steepness is $(a k)_{0}=0.13, q_{c}$ lies in $\mathscr{I}\left(\frac{1}{2}\right)$ while for higher steepness $q_{c}$ lies in $\mathscr{I}(0)$. The experimental results show moreover that the transition from $L_{2}$ to $L_{1}$ patterns with increasing steepness is accompanied by a transition from $q_{c}=n k_{\perp}$ to $q_{c}=(n-1) k_{\perp}$ (see figure $1 c, d$ of Collard \& Caulliez, with $n=8$ ). As emphasized in $\S 6.2 .2$, the plastic film has the effect of bringing the instability diagram closer to the $p$-axis than theoretically expected without it. We hence cannot simulate numerically exactly the experiments with the plastic film and we shall assume that its unique effect is to cancel modulational instability. Hence we do not expect to reproduce the experimentally observed value of $q_{c}$. The simulated growth rate is expected to be quite different from the observations. The selection mechanism suggested here is assumed to work for both numerical simulations and experiments, nevertheless.

We thus consider condition (i) to be fulfilled and we numerically suppress development of modulational instability. Having computed the margin of stability for the first cross-waves in this case, we showed above that parametric resonance was possible and that condition (ii) was fulfilled.

Figure 26 shows the instability diagrams for two values of the steepness $(a k)_{0}=0.13$ and $(a k)_{0}=0.17$. It is clear that in this case condition (iii) is fullfiled in the whole range of wave steepness considered experimentally.

Assume now a tank width $b$ such that there exists only one multiple of $k_{\perp}$ which falls into the class II unstable region and is included in $\mathscr{I}\left(\frac{1}{2}\right)$. There exists then an integer $n$ such that $q_{c}=n k_{\perp}$ belongs to $\mathscr{I}\left(\frac{1}{2}\right): L_{2}$ patterns are observed. When the wave steepness increases, the instability region is moved closer to the $p$-axis. There exists a critical steepness for which $n k_{\perp}$ is no longer included in $\mathscr{I}\left(\frac{1}{2}\right)$, while $(n-1) k_{\perp} \in \mathscr{I}(0)$ : $L_{1}$ patterns are observed. Inversely, considering two values of the steepness, one may choose the tank width $b$ in such a way that this transition is possible. Figure 26 also shows two consecutive eigenmodes of the channel which have been chosen for the 
numerical simulations. In this case the previously described scenario can be applied and such a transition between the two values of the steepness is possible.

\subsection{Comments on extension to finite depth}

Generalization of the previous result should be to consider finite-depth simulations. We have already pointed out in $\S 5$ that in the finite-depth case the class I and class II instabilities have comparable strengths for moderately steep waves if $(a k)_{0}$ is around 0.1 , which corresponds to $30 \%$ of the highest steepness. Hence conditions (i) and (iii) are fulfilled if moderately steep waves are considered. In a channel of finite depth, the parametric excitation is still valid and theoretical analysis is known to lead to similar results (with only a small correction due to the finite-depth effect). It Would be interesting to reproduce in a wave laboratory the experiments of Collard \& Caulliez (1999) on oscillating patterns, in finite depth. In this case a plastic film is not necessary to generate $L_{1}$ patterns.

\section{Conclusion}

A detailed investigation of three-dimensional patterns occurring at the sea surface has been presented. Their study is important to provide a better description of the sea surface roughness which is crucial to determine correctly fluxes between ocean and atmosphere. The time scales during which these three-dimensional structures can be observed depend on the steepness of the initial Stokes wave trains. Their nonlinear evolution usually results in the breaking of the wave.

Before investigating wave trains evolving to breaking, we checked that the numerical method was able to treat very steep gravity waves. A Stokes wave train with a steepness of $(a k)_{0}=0.4$ was propagated steadily for 100 periods. The efficiency of the Eulerian numerical approach used herein to recognize waves evolving to breaking has been checked in two-dimensional and extension to a three-dimensional free surface has been developed. Numerical simulations taking into account both class I and class II instabilities showed that for moderately steep waves, namely $(a k)_{0}>0.12$, their nonlinear coupling (involving the fundamental of the Stokes wave) results in breaking of the wave when in the initial condition only the modulational instability was considered. This result is in agreement with the experiments conducted by $\mathrm{Su} \&$ Green (1985). Furthermore, we found that breaking can occur for $(a k)_{0}=0.10$ when the initial unstable perturbation corresponds to the phase-locked crescent-shaped patterns. At the maximum amplitude of this instability the modulational instability is excited followed by the breaking of the wave. For steeper waves, the strength of class II instability alone is sufficient to trigger breaking of the wave. It was shown that the nonlinear dynamics of the most unstable class II perturbation leads to breaking when $(a k)_{0}>0.17$.

For moderately steep waves, deep water modulational instability occurs naturally. To observe the oscillating horseshoe patterns in a water wave tank of infinite depth Collard \& Caulliez (1999) used a plastic film to cancel this instability. It was shown that parametric resonances due the wave-maker could select and amplify the occurrence of the oscillating patterns observed in water wave tanks. Despite this artificial generation we believe that the $L_{1}$ patterns may be observed in coastal zones where the strength of modulational instability is attenuated. It would be of primary interest to conduct experiments in a tank of finite depth to check the observability of these three-dimensional structures. 
This work was conducted under the BmatA-programme 'Computational methods for stratified flows involving internal waves' and the Strategic University Programme 'Modelling of currents and wave for sea structures', both funded by the Research Council of Norway. The authors would like as well to express their gratitude to the referees for their helpful comments.

\section{REFERENCES}

AnNenkov, S. Y. \& ShriRA, V. I. 2001 Numerical modelling of water-waves evolution based on the Zakharov equation. J. Fluid Mech. 449, 341-372.

BANNER, M. L. \& TiAn, X. 1996 Energy and momentum growth rates in breaking water waves. Phys. Rev. Lett. 77, 2953-2956.

BANnER, M. L. \& TiAn, X. 1998 On the determination of the onset of breaking for modulating surface gravity water waves. J. Fluid Mech. 367, 107-137.

Benjamin, T. B. \& Feir, J. E. 1967 The desintegration of wave trains on deep water. Part. 1. Theory. J. Fluid Mech. 27, 417-430.

Chen, G., Kharif, C., Zaleski, S. \& Li, J. 1999 Two-dimensional Navier-Stokes Simulation of Breaking Waves. Phys. Fluids 11, 121-133.

Clamond, D. \& Grue, J. 2001 A fast method for fully nonlinear water wave computations. J. Fluid Mech. 447, 337-355.

Collard, F. \& Caulliez, G. 1999 Oscillating crescent-shaped water wave patterns. Phys. Fluids, Lett. 11, 3195-3197.

Craig, W. 2001 On the Badullin, Kharif and Shrira model of resonant water waves. Physica D 152-153, 434-450.

Dias, F. \& KhaRIF, C. 1999 Nonlinear gravity and capillarity-gravity waves. Annu. Rev. Fluid Mech. 31, 301-341.

Dold, J. W. \& Peregrine, D. H. 1986 Water wave modulation. Proc. 20th Intl Conf. Coastal Engng, Taipei, ASCE 1, pp. 163-175

Dommermuth, D. \& Yue, D. K. P. 1987 A high-order spectral method for the study of nonlinear gravity waves. J. Fluid Mech. 184, 267-288.

Fenton, J. D. 1988 The numerical solution of steady water wave problems. Computers Geosci. 14, 357-368.

Fructus, D., Clamond, D., Grue, J. \& Kristiansen, Ø. 2005 Efficient numerical model for three-dimensional gravity waves simulations. Part I: Periodic domains. J. Comput. Phys. 205, 665-685.

Francius, M. \& KHARIF, C. 2005 Three-dimensional instability of nonlineargravity waves in shallow water. J. Fluid Mech. (submitted).

Fuhrman, D. R., Madsen, P. A. \& Bingham, H. B. 2004 A numerical study of crescent waves. J. Fluid Mech. 513, 309-342.

Garrett, C. J. R. 1970. On cross-waves. J. Fluid Mech. 41, 837-849.

GRUE, J. 2002 On four highly nonlinear phenomena in wave theory and marine hydrodynamics. Appl. Ocean Res. 24, 261-274.

JONES, F. 1984 The generation of cross-waves in a long deep channel by parametric resonance. J. Fluid Mech. 138, 53-74.

Kharif, C. \& Pontier, R. 2000 Occurrence of new 3d patterns in water wave fields. Proc. XXth ICTAM, Chicago.

Kharif, C. \& Ramamonjiarisoa, A. 1988 Deep-water gravity waves instabilities at large steepness. Phys. Fluids 31, 1286-1288.

Kharif, C. \& Ramamonjiarisoa, A. 1990 On the stability of gravity waves on deep water. J. Fluid Mech. 218, 163-170.

Kusuba, T. \& Mitsuyasu, M. 1986 Nonlinear instability and evolution of steep water waves under wind action. Rep. Res. Inst. Appl. Mech. Kyushu Univ. 33(101), 33-64.

Lichter, S. \& Chen, J. 1987 Subharmonic resonance of nonlinear cross-waves. J. Fluid Mech. 183, 451-465.

Lighthill, M. J. 1965 Contributions to the theory of waves in nonlinear dispersive systems J. Inst. Math. Applics. 1, 269-306. 
Longuet-Higgins, M. S. 1978 The instabilities of gravity waves of finite amplitude in deep water. II. Subharmonics Proc. R. Soc. Lond. A 360, 489-505.

Longuet-Higgins, M. S. 1985 Bifurcations in gravity waves. J. Fluid Mech. 151, 457-475.

Mahony, J. J. 1972 Cross-waves. Part 1. Theory. J. Fluid Mech. 55, 229-244.

MCLEAn, J. W. $1982 a$ Instability of finite amplitude water waves. J. Fluid Mech. 114, 315-330.

MCLEAN, J. W. $1982 b$ Instability of finite amplitude water waves on water of finite depth. J. Fluid Mech. 114, 331-341.

Mclean, J. W., Ma, Y. C., Martin, D. U., Saffman, P. G. \& Yuen, H. C. 1981 Three dimensional instability of finite amplitude water waves. Phys. Rev. Lett. 46, 817-820.

Meiron, D. I., Saffman, P. G. \& Yuen, H. C. 1982 Calculation of steady three-dimensional deep-water waves. J. Fluid Mech. 124, 109-121.

Melville, W. K. 1982 The instability and breaking of deep-water waves. J. Fluid Mech. 115, 165-185.

MiLEs, J. 1988 Parametrically excited, standing cross-waves. J. Fluid Mech. 186, 119-127.

Miles, J. \& Becker, J. 1988 Parametrically excited, progressive cross-waves. J. Fluid Mech. 186, $129-146$.

Miles, J. \& Henderson, D. 1990 Parametrically forced surface waves. Annu. Rev. Fluid Mech. 22, $143-165$.

Phillips, O. M. 1960 On the dynamics of unsteady gravity waves of finite amplitude. J. Fluid Mech. 9, $193-217$.

Shrira, V. I., Badulin, S. I. \& Kharif, C. 1996 A model of water wave 'horse-shoe' patterns. J. Fluid Mech. 318, 375-404.

SKandrani, C. 1996 Contribution à l'étude de la dynamique non linéaire des champs de vagues tridimensionnels en profondeur infinie, $\mathrm{PhD}$ thesis, Université de la Méditerranée.

Stiassnie, M. \& Shemer, L. 1987 Energy computations for evolution of class I and II instabilities of Stokes waves. J. Fluid Mech. 174, 299-312.

Su, M. Y. 1982 Three-dimensional deep-water waves. Part 1. Experimental measurement of skew and symetric wave patterns. J. Fluid Mech. 124, 73-108.

Su, M. Y., Bergin, M., Marler, P. \& Myrick, R. 1982 Experiments on nonlinear instabilities and evolution of steep gravity-wave trains. J. Fluid Mech. 124, 45-72.

Su, M. Y. \& GreEN, A. W. 1984 Coupled two- and three-dimensional instabilities of surface gravity waves. Phys. Fluids 27, 2595-2597.

Su, M. Y. \& GReEN, A. W. 1985 Wave breaking and nonlinear instability coupling. In The ocean Surface: Wave breaking, Turbulent Mixing and Radio Probing (ed. Y. Toba \& H. Mitsuyasu). D. Reidel.

Xue, M., Xü, H., LiU, Y. \& Yue, D. K. P. 2001 Computations of fully nonlinear three-dimensional wave-wave and wave-body interactions. Part 1 . Dynamics of steep three-dimensional waves. J. Fluid Mech. 438, 11-39.

ZaKharov, V. E. 1968 Stability of periodic waves of finite amplitude on the surface of a deep fluid. J. Appl. Mech. Tech. Phys. 9, 190-194. 